TRANSACTIONS OF THE

AMERICAN MATHEMATICAL SOCIETY

Volume 348, Number 7, July 1996

\title{
PROPERTIES OF EXTREMAL FUNCTIONS FOR SOME NONLINEAR FUNCTIONALS ON DIRICHLET SPACES
}

\author{
ALEC MATHESON AND ALEXANDER R. PRUSS
}

\begin{abstract}
Let $\mathfrak{B}$ be the set of holomorphic functions $f$ on the unit disc $D$ with $f(0)=0$ and Dirichlet integral $(1 / \pi) \iint_{D}\left|f^{\prime}\right|^{2}$ not exceeding one, and let $\mathfrak{b}$ be the set of complex-valued harmonic functions $f$ on the unit disc with $f(0)=0$ and Dirichlet integral $(1 / 2)(1 / \pi) \iint_{D}|\nabla f|^{2}$ not exceeding one. For a (semi)continuous function $\Phi:[0, \infty) \rightarrow \mathbb{R}$, define the nonlinear functional $\Lambda_{\Phi}$ on $\mathfrak{B}$ or $\mathfrak{b}$ by $\Lambda_{\Phi}(f)=\frac{1}{2 \pi} \int_{0}^{2 \pi} \Phi\left(\left|f\left(e^{i \theta}\right)\right|\right) d \theta$. We study the existence and regularity of extremal functions for these functionals, as well as the weak semicontinuity properties of the functionals. We also state a number of open problems.
\end{abstract}

\section{INTRODUCTION}

Let $\mathfrak{D}$ be the (holomorphic) Dirichlet space of functions $f$ holomorphic on the unit disc $D=\{z \in \mathbb{C}:|z|<1\}$, with $f(0)=0$ and

$$
\|f\|_{\mathfrak{D}}^{2} \stackrel{\text { def }}{=} \frac{1}{\pi} \iint_{D}\left|f^{\prime}(x+i y)\right|^{2} d x d y<\infty .
$$

Equipped with the inner product

$$
(f, g) \stackrel{\text { def }}{=} \frac{1}{\pi} \iint_{D} f^{\prime}(x+i y) \overline{g^{\prime}(x+i y)} d x d y
$$

$\mathfrak{D}$ becomes a Hilbert space. Let $\mathfrak{B}=\left\{f \in \mathfrak{D}:\|f\|_{\mathfrak{D}} \leq 1\right\}$ be its closed unit ball. Note that it is easy to verify that

$$
\|f\|_{\mathfrak{D}}^{2}=\sum_{n=1}^{\infty} n|\hat{f}(n)|^{2}
$$

and

$$
(f, g)=\sum_{n=1}^{\infty} n \hat{f}(n) \overline{\hat{g}}(n)
$$

Received by the editors September 8, 1994 and, in revised form, September 5, 1995.

1991 Mathematics Subject Classification. Primary 30A10, 30D99; Secondary 28A20, 49J45, $49 \mathrm{~K} 99$.

Key words and phrases. Dirichlet space, Dirichlet integral, Beurling's estimate, convergence in measure, Chang-Marshall inequality, harmonic majorants and rearrangement, optimization problems, necessary conditions for extremality, regularity of extremals.

The research of the second author was partially supported by Professor J. J. F. Fournier's NSERC Grant \#4822. Portions of this paper also appear in a part of the second author's doctoral dissertation. 
where $\hat{f}(n)$ denotes the $n$th Taylor coefficient of $f$. It is useful for purposes of intuition to note that $\pi\|f\|_{\mathfrak{D}}^{2}$ is the area of the image $f(D)$, counting multiplicities.

To place $\mathfrak{D}$ in a larger picture, it should be noted that $\mathfrak{D}$ contains unbounded functions (any univalent map to an unbounded region of finite area will be in $\mathfrak{D}$ ), and that not every function from the disc algebra lies in $\mathfrak{D}$. This last fact is easiest proved by choosing a summable lacunary sequence of Taylor coefficients for which (0.2) fails be to be finite. Finally, $\mathfrak{D} \subset$ BMOA. One of the easier ways to prove this is to note that from $(0.2)$ it follows that if $f \in \mathfrak{D}$ then

$$
\sup _{m \geq 1} \sum_{n=1}^{\infty}\left(\sum_{j=1}^{m-1}|\hat{f}(m n+j)|\right)^{2}<\infty,
$$

which is C. Fefferman's sufficient (and, if we have $\hat{f}(k) \geq 0$ for all $k \geq 0$, then also necessary) condition for a holomorphic function $f$ on $D$ to be a member of BMOA (see, e.g., [21] or [4]).

In this paper we shall be concerned with functionals $\Lambda_{\Phi}$ on $\mathfrak{B}$ defined by

$$
\Lambda_{\Phi}(f)=\int_{\mathbb{T}} \Phi(|f|),
$$

for $f \in \mathfrak{B}$ and $\Phi:[0, \infty) \rightarrow \mathbb{R}$ having various (semi)continuity properties, where $\mathbb{T}$ is the unit circle with normalized Lebesgue measure, so that $\int_{\mathbb{T}} \Phi(|f|)$ is short for $\frac{1}{2 \pi} \int_{0}^{2 \pi} \Phi\left(\left|f\left(e^{i \theta}\right)\right|\right) d \theta$.

These questions are motivated by the Chang-Marshall inequality which states that if we put

$$
\Phi_{\alpha}(t)=e^{\alpha t^{2}},
$$

for positive $\alpha$, then $\Lambda_{\Phi_{\alpha}}$ is bounded on $\mathfrak{B}$ if and only if $\alpha \leq 1$. Given an estimate of Beurling [3] which we shall discuss later, the cases $\alpha \neq 1$ are not difficult to handle. Chang and Marshall's original proof [7] of the boundedness for $\alpha=1$ used arguments based on ideas from the proof an inequality of J. Moser [17]. A shorter potential-theoretic proof which uses Moser's inequality directly has since been found by Marshall [15]. On the other hand, Essén [10] has found a significantly more general and stronger inequality. Among other things he shows that $\Lambda_{\Phi_{1}}(f)$ remains uniformly bounded in $f$ if the condition $\|f\|_{\mathfrak{D}} \leq 1$ is weakened to the condition that the area of the range $f(D)$, not counting multiplicities, does not exceed $\pi$ (recall that $\pi\|f\|_{\mathfrak{D}}^{2}$ was the area, counting multiplicities).

We shall show that the Chang-Marshall inequality is so sharp that we cannot replace $\Phi_{1}(t)=e^{t^{2}}$ by $\phi(t) e^{t^{2}}$ for any non-negative $\phi$ tending to infinity. More precisely, we have the following result.

Theorem 1. Suppose $\Phi(t)=\phi(t) e^{t^{2}}$ has $\Lambda_{\Phi}$ bounded on $\mathfrak{B}$, or even just on the Beurling functions, for a non-negative $\phi$. Then $\phi(t)$ cannot converge to $\infty$ as $t \rightarrow \infty$. In particular, if $\phi$ is non-decreasing, then it must be bounded.

The Beurling functions $B_{a}$ are defined by

$$
B_{a}(z)=\frac{\log \frac{1}{1-\bar{a} z}}{\sqrt{\log \frac{1}{1-|a|^{2}}}},
$$


for $a \in D \backslash\{0\}$, where the branch of the logarithm is chosen so that $B_{a}(a)$ is real. The denominator was chosen so that $\left\|B_{a}\right\|_{\mathfrak{D}}=1$. Chang and Marshall's original proof [7] of their inequality proceeded by first proving it for the Beurling functions, and then comparing general functions to them. Up to a normalizing factor, the $B_{a}$ are the kernel functions for $\mathfrak{D}$.

The final result which we need about the Dirichlet space is Beurling's estimate, which we have already mentioned. Let

$$
E_{M}(f)=|\{z \in \mathbb{T}:|f(z)| \geq M\}| .
$$

Note that these level functions fit into our general setting, since $E_{M}(f)=\Lambda_{\Phi}(f)$, where $\Phi$ is the indicator function of the interval $[M, \infty)$. Beurling proved in his influential thesis [3, pp. 34-37] that

$$
E_{M}(f) \leq e^{1-M^{2}}
$$

whenever $f \in \mathfrak{B}$. He also showed [3, pp. 39-41] that this estimate is sharp in the sense that there is an absolute constant $c>0$ such that

$$
E_{M}\left(B_{a}\right) \geq c e^{-M^{2}},
$$

when

$$
M=\sqrt{\log \frac{1}{1-|a|^{2}}} .
$$

From (0.6) it is not hard to show that if $p$ is any polynomial and $C$ any finite constant, then

$$
p(|f|) e^{C|f|^{2}} \in L^{r}(\mathbb{T}),
$$

for every $f \in \mathfrak{D}$ and every finite $r$ (see, e.g., [7], where this observation is attributed to J. Garnett). Furthermore, as mentioned before, it is well known (and the knowledge of this fact preceded the Chang-Marshall inequality) that one can use Beurling's estimate to show that $\Lambda_{\Phi_{\alpha}}$ is bounded for $\alpha<1$, by using the expression

$$
\int_{\mathbb{T}} e^{\alpha|f|^{2}}=1+2 \alpha \int_{0}^{\infty} E_{M}(f) M e^{\alpha M^{2}} d M
$$

Definition. A function $f$ is extremal for a functional $F$ defined on a set of functions $S$ provided $f \in S$ and $F(f) \geq F(g)$ for all $g \in S$. In particular, a function $f$ is extremal for $\Lambda_{\Phi}$ if $f \in \mathfrak{B}$ and $\Lambda_{\Phi}(f) \geq \Lambda_{\Phi}(g)$ for all $g \in \mathfrak{B}$.

In the first section of this paper we give results concerning existence of extremal functions $f \in \mathfrak{B}$ for the functional $\Lambda_{\Phi}$ for certain classes of functions $\Phi:[0, \infty) \rightarrow \mathbb{R}$, using weak semicontinuity methods. Theorem 1 will follow from a result given in that section. Some of our results are general propositions concerning convergence in measure on a finite measure space, and are related to the direct methods in the calculus of variations. In the second section we discuss the generalization of the results of the first section from the holomorphic Dirichlet space to various harmonic Dirichlet spaces. In particular, we use the Chang-Marshall inequality to obtain an analogous inequality which is maximally sharp in the sense of Theorem 1 for the complex-valued harmonic Dirichlet space. We also discuss the generalization to non-radially-symmetric functionals.

In the third section we discuss the regularity of the extremal functions. In particular we demonstrate a more general version of the following result. 
Theorem 2. Suppose $\Phi:[0, \infty) \rightarrow \mathbb{R}$ is such that $\Phi(\sqrt{t})$ is infinitely differentiable with respect to $t$ on $(0, \infty)$ and each of its derivatives is bounded near zero. Also assume that $\Phi^{\prime}(t) \neq 0$ on $(0, \infty)$, and that there is a finite constant $C$ such that $\left|\Phi^{\prime}(t)\right|=O\left(e^{C t^{2}}\right)$ as $t \rightarrow \infty$. Then any extremal function for $\Lambda_{\Phi}$ must lie in $C^{\infty}(\bar{D})$, i.e., for every non-negative $k$ we must have $f^{(k)}$ in the disc algebra.

Note. The theorem does not assert that there actually exists an extremal for $\Lambda_{\Phi}$, but only that if there is one, then it must be in $C^{\infty}(\bar{D})$.

In the fourth section we discuss generalizations of Theorem 2 and similar theorems for various harmonic cases and for the case where the functionals are not radially-symmetric. A particular example of this is the following result.

Theorem 3. Suppose that $\Phi: \mathbb{R}^{2} \rightarrow \mathbb{R}$ is infinitely differentiable, and that the first partials $\Phi_{, j}$ satisfy the condition $\left|\Phi_{, j}\right|=O\left(e^{C t^{2}}\right)$, for $j=1,2$ and some constant $C$. Assume also that $\Phi$ satisfies the strict monotonicity condition $x_{1} \Phi_{, 1}\left(x_{1}, x_{2}\right)+$ $x_{2} \Phi_{, 2}\left(x_{1}, x_{2}\right)>0$ for $\left(x_{1}, x_{2}\right) \neq(0,0)$. Then if the functional $f \mapsto \int_{\mathbb{T}}(\Phi \circ f)$, defined on the set of complex-valued harmonic functions $f$ on $D$ with $f(0)=0$ and Dirichlet integral $\frac{1}{2 \pi} \iint_{D}|\nabla f|^{2}$ not exceeding one, has an extremal function, then this extremal function must lie in $C^{\infty}(\bar{D})$.

Note that one can actually drop the requirement of harmonicity of $f$ here and consider all functions $f$ on $D$ which lie in the Sobolev space $C(D) \cap W_{2}^{1}(D)$, and have Dirichlet integrals not exceeding 1 and whose boundary values (defined via the operator coming from the trace theorem) have mean zero. For, given such an $f$, assuming it is not harmonic, by a generalization of the Dirichlet principle there will be a harmonic $g$ whose Dirichlet integral will be strictly smaller, but whose boundary values will be the same, and by our strict monotonicity condition we will be able to increase the functional by renormalizing $g$, so that $f$ cannot be non-harmonic. Thus the extremals are automatically harmonic.

In the fifth and final section we give the proofs of the more difficult theorems from the first, second and third sections in cases where we have judged it expedient not to give them immediately.

Finally, we mention the following open problem which we feel is quite interesting, but which we shall not discuss beyond this section except for a brief mention in $\S 3$. More information can be found in [18].

Open Problem 1. Let $U$ be a domain in the complex plane with finite area, containing the origin. Let $\mathcal{F}$ be the collection of $\Phi:[0, \infty) \rightarrow \mathbb{R}$ such that $\Phi\left(e^{t}\right)$ is convex and non-decreasing in $t$; we will then have $\Phi(|z|)$ subharmonic on $U$. For $\Phi \in \mathcal{F}$, let $h(z)$ be the least harmonic majorant for $\Phi(|z|)$ on $U$, and set $\Gamma_{\Phi}(U)=h(0)$.

(i) Is there a simply connected domain $\tilde{U}$ (independent of $\Phi$ ) such that Area $(\tilde{U})$ $\leq \operatorname{Area}(U)$ and $\Gamma_{\Phi}(\tilde{U}) \geq \Gamma_{\Phi}(U)$ for every $\Phi \in \mathcal{F}$ ?

(ii) Can, moreover, $\tilde{U}$ be taken to be starlike?

In the notation of this problem, Essén's result [10] mentioned above says that if $\operatorname{Area}(U) \leq \pi$ and $\Phi_{1}(t)=e^{t^{2}}$ then $\Gamma_{\Phi_{1}}(U) \leq C$ for some absolute constant $C$.

Whenever $U$ is simply connected and $\Phi \in \mathcal{F}$, then $\Gamma_{\Phi}(U)=\Lambda_{\Phi}(F)$, where $F$ is a Riemann map from $D$ onto $U$ with $F(0)=0$. To prove this we first note that if $\Gamma_{\Phi}(U)<\infty$ and $h$ is the least harmonic majorant for $\Phi(|\cdot|)$ on $U$ then, as in [10, 
Proof of Cor. 1], we have

$$
\int_{0}^{2 \pi} \Phi\left(\left|F\left(r e^{i \theta}\right)\right|\right) d \theta \leq \int_{0}^{2 \pi} h\left(F\left(r e^{i \theta}\right)\right) d \theta=2 \pi h(F(0))=2 \pi h(0),
$$

for every $0 \leq r<1$. Taking the limit as $r \uparrow 1$ we conclude that $\Lambda_{\Phi}(F) \leq \Gamma_{\Phi}(U)$. Assuming $\Lambda_{\Phi}(F)<\infty$, the converse inequality then follows from the fact that $F$ lies in the Nevanlinna subclass $N^{+}$(since $F$ is univalent), so that, as in [11, p. 515],

$$
\lim _{r \rightarrow 1-} \frac{1}{2 \pi} \int_{0}^{2 \pi} \Phi\left(\left|F\left(r e^{i \theta}\right)\right|\right) d \theta=\frac{1}{2 \pi} \int_{0}^{2 \pi} \Phi\left(\left|F\left(e^{i \theta}\right)\right|\right) d \theta=\Lambda_{\Phi}(F),
$$

and then, as usual, if $u_{r}$ is the least harmonic majorant of $\Phi(|F|)$ on the disc $\{|z|<r\}$, where $0 \leq r<1$, we will have $\sup _{0 \leq r<1} u_{r}(0)=\Lambda_{\Phi}(F)<\infty$, since $u_{r}(0)$ is equal to the integral on the left hand side of (0.11), and, letting $r \uparrow 1$, Harnack's principle implies that the $u_{r}$ converge to a harmonic majorant for $\Phi(|F|)$ on $D$, whose value at zero will be precisely $\Lambda_{\Phi}(F)$ (cf. [12, Ch. I, Sec. 6]). A similar result of Burkholder and Baernstein can be found in [5].

Thus, if (i) were to hold, then Essén's result would be a consequence of the Chang-Marshall inequality (indeed, of the special case where the Chang-Marshall inequality is only shown for univalent functions.) It would also follow that to obtain the supremum of $\Lambda_{\Phi}$ over $\mathfrak{B}$, where $\Phi \in \mathcal{F}$, it would suffice to test univalent (and starlike if (ii) also has an affirmative answer) functions. Furthermore, if such a $\Lambda_{\Phi}$ achieved its maximum on $\mathfrak{B}$, then it would achieve it on the univalent (respectively, starlike) functions, if the answer to (i) or, respectively, to (ii), were affirmative.

We also remark that a positive answer to Problem 1(i) would, together with the fact due to Andreev and Matheson [1] that the $L^{p}(\mathbb{T})$ norms for finite $p$ are weak continuous on $\mathfrak{D}$ so that $\Lambda_{\Phi}$ for $\Phi(t)=t^{p}$ has an extremal function because of the weak compactness of $\mathfrak{B}$, would imply a positive answer to a problem of Sakai [20] as to whether there exist extremal domains $U$ with Area $U=\pi$ for the functional $\Gamma_{\Phi}$ where $\Phi(t)=t^{p}$. (See Sakai [20, Thm. 5.10] for a positive solution of the analogous problem in all dimensions other than 2.)

We note that circular rearrangement does increase the $\Gamma_{\Phi}$. This follows from [2, Thm. 5], though one can also prove it directly by modifying the proof of $[2$, Thm. 7]. Marcus [14] has studied a radial rearrangement method which does not increase area, while it does increase some capacities and transforms domains containing the origin into starshaped ones. Unfortunately, there exist very simple counterexamples where this rearrangement method decreases some of the $\Gamma_{\Phi}$. Recently, however, the second author has conjectured that Marcus' rearrangement does not decrease the $\Gamma_{\Phi}$ if it is applied to a circularly symmetric domain. Were this conjecture just, an affirmative answer to Problem 1 would follow by combining Marcus' radial rearrangement with circular symmetrization. See [18] for the counterexamples mentioned above and for further information on the problem.

\section{EXISTEnCE OF EXTREMAL FUnCTIONS}

The main tools for results in this section are the weak compactness of $\mathfrak{B}$ and the fact that weak convergence in $\mathfrak{B}$ implies convergence in measure on $\mathbb{T}$. Indeed, as shown in [1], weak convergence in $\mathfrak{B}$ implies convergence in $L^{p}(\mathbb{T})$, for $0<p<\infty$, 
which in turn implies convergence in measure. If we can show that a functional is weak upper semicontinuous on $\mathfrak{B}$ then we will be able to immediately conclude that it achieves its maximum there.

The authors would like to encourage the reader to review the work of Andreev and Matheson [1] (and perhaps also that of Cima and Matheson [8]) to make clearer the exact nature of the present work.

The outstanding open problem in this area is the following.

Open Problem 2. Does the Chang-Marshall functional $\Lambda_{\Phi_{1}}$, where $\Phi_{1}(t)=e^{t^{2}}$, have an extremal function?

It is known that restricted to the closed convex hull of the Beurling functions it attains its maximum [8], and it was conjectured by Andreev and Matheson [1] that it attains its maximum on $\mathfrak{B}$ at the identity function. This last conjecture is supported by the second author's numerical verification of the inequality $\Lambda_{\Phi_{1}}(p) \leq e$ (since $e$ is the value of $\Lambda_{\Phi_{1}}$ at the identity function) for over 40 million pseudorandomly chosen polynomials $p$ of degree 6 . However, the general methods of the present paper cannot be used to give the existence of an extremal function for $\Lambda_{\Phi_{1}}$, because all the methods herein listed are based on upper semicontinuity, and $\Lambda_{\Phi_{1}}$ fails to be upper semicontinuous at zero (see [8, end of proof of Thm. 1], or the proof of Theorem 1 given below). Thus the problem remains open. We note that Carleson and Chang [6] have shown that extremal functions do exist in the closely related inequality of Moser [17] (see also [16] for another proof of this non-trivial result).

Finally, we remark that it has been very recently shown $[19$, Thm. 5] that there exists a perturbation $\tilde{\Phi}$ of $\Phi_{1}$ such that $\Lambda_{\tilde{\Phi}}$ has no extremals over $\mathfrak{B}$ even though $\tilde{\Phi}$ is a non-negative non-decreasing convex infinitely differentiable function with $\tilde{\Phi}(t) \leq e^{t^{2}}$ and $\lim _{t \rightarrow \infty} e^{-t^{2}} \tilde{\Phi}(t)=1$. However, this does not provide any evidence against Problem 2 since an analogous result has also been shown in [19, Thm. 4] to hold with regard to the Moser inequality, despite the fact that an extremal is known to exist in the latter. Moreover, it is not very difficult to show that there exists an infinitely differentiable function $\Psi$ which agrees with $\Phi_{1}$ outside of some bounded interval $[0, T]$ such that $\Lambda_{\Psi}$ has an extremal over $\mathfrak{B}$ (see [19, Thm. 5]).

As noted above, weak convergence in $\mathfrak{B}$ implies convergence in measure on $\mathbb{T}$. Thus, we first give some results on convergence in measure, which immediately specialize to give results concerning weak semicontinuity on the unit ball of the Dirichlet space, or indeed semicontinuity on any separable topological space of functions on a finite measure space whose topology is at least as fine as convergence in measure. (To avoid confusion, we should note that we say that a topology $\tau$ is at least as fine as a topology $\tau^{\prime}$ if all $\tau^{\prime}$-open sets are also $\tau$-open.)

Let $(I, \mu)$ be a finite measure space. As before, but now in a more general setting, write

$$
\Lambda_{\Phi}(f)=\int_{I} \Phi(|f|) d \mu .
$$

The following result is a direct consequence of Fatou's lemma.

Lemma 1. Let $\Phi:[0, \infty) \rightarrow[0, \infty)$ be lower semicontinuous. Then, given a sequence $\left\{f_{n}\right\}$ of measurable functions on I converging in measure to $f$, we must have

$$
\liminf _{n \rightarrow \infty} \Lambda_{\Phi}\left(f_{n}\right) \geq \Lambda_{\Phi}(f) .
$$


We then obtain the following simple theorem.

Theorem 4. Let $B$ be a set of measurable functions on $I$ equipped with some separable topology $\tau$ at least as fine as convergence in measure. Let $\Phi:[0, \infty) \rightarrow \mathbb{R}$ be upper semicontinuous and let $\Psi:[0, \infty) \rightarrow \mathbb{R}$ be lower semicontinuous with $\Phi \leq \Psi$ on $[0, \infty)$. Then if $\Lambda_{\Psi}$ is upper semicontinuous on $B$ with respect to $\tau$, so is $\Lambda_{\Phi}$.

Proof. Let $f_{n} \rightarrow f$ be a $\tau$-convergent sequence in $B$. This sequence must also converge in measure. But $\Psi-\Phi$ is lower semicontinuous and nonnegative, so that by Lemma 1 we have $\liminf _{n \rightarrow \infty} \Lambda_{\Psi-\Phi}\left(f_{n}\right) \geq \Lambda_{\Psi-\Phi}(f)$, and thus $\Lambda_{\Psi-\Phi}$ is $\tau$-lower semicontinuous on $B$. But $\Lambda_{\Phi}=\Lambda_{\Psi}-\Lambda_{\Psi-\Phi}$, and $\Lambda_{\Psi}$ is $\tau$-upper semicontinuous, so $\Lambda_{\Phi}$ must be $\tau$-upper semicontinuous as well.

Thus if we take $I=\mathbb{T}$ and $B=\mathfrak{B}$ with the weak topology, then under the conditions of the theorem, $\Lambda_{\Phi}$ is weak upper semicontinuous on $\mathfrak{B}$, and hence it will attain its maximum there.

Corollary 1. The functionals $E_{M}$ defined by (0.5) for $M \geq 0$ are weak upper semicontinuous on $\mathfrak{B}$ and hence attain their maxima there.

Proof. Let $\Phi(t)$ be the indicator function of $[M, \infty)$ so that $E_{M}=\Lambda_{\Phi}$, and let $\Psi(t) \equiv 1$. Then $\Phi$ is upper semicontinuous, while clearly $\Psi$ is lower semicontinuous and $\Lambda_{\Psi}$ is upper semicontinuous, so that our result follows from the trivial inequality $\Phi \leq \Psi$ together with Theorem 4.

We do not know any specific functions $f \in \mathfrak{B}$ that maximize the $E_{M}(f)$ if $M>1$, but the above result implies they exist.

Open Problem 3. Find functions that maximize the $E_{M}$ if $M>1$.

The extremals are not expected to be unique, not even up to a multiplicative constant of modulus one. If $M \leq 1$, then, e.g., any univalent map $f$ whose range has area at most $\pi$ and includes the disc $\{z:|z|<M\}$ will maximize $E_{M}(f)$ (with the maximum $E_{M}(f)=1$ ), and there are many such $f$ if $M<1$.

Open Problem 4. Determine the extremal functions for other simple choices of $\Phi$, such as $\Phi(t)=t^{2 n}$.

This last choice does lead to the existence of extremal functions (since the $L^{p}(\mathbb{T}$ ) norms for finite $p$ are weak continuous on $\mathfrak{D}$ by [1]), and, by Theorem 2 , the extremal functions will in fact have to lie in $C^{\infty}(\bar{D})$. It is easy to see that the identity function is extremal for $\Phi(t)=t^{2}$, and the first author has shown it to be extremal for $t^{p}$ whenever $0<p \leq 4$. (Professor Makoto Sakai has kindly informed the authors that he had heard of Professors N. Suita and S. Kobayashi independently proving almost the very same fact.) However, it is not known what the extremal functions for $\Phi(t)=t^{2 n}$ look like for $n>2$. As Andreev and Matheson [1] note, for $n$ sufficiently large the identity function will not be extremal, since $\mathfrak{B}$ contains unbounded functions. In fact, the following result holds.

Proposition. (Sakai [20]) If $\Phi(t)=t^{p}$ where $p>4$, then there exists $f \in \mathfrak{B}$ such that $\Lambda_{\Phi}(f)>\Lambda_{\Phi}(z)$, where $z$ indicates the identity function.

Proof. Let $\Phi(t)=t^{p}$ for some $p>4$. Let $\Gamma_{\Phi}$ be the functional in Problem 1. Let $D_{w}$ be an open disc of unit radius centred about the point $w$. The proof of $[20$, 
Prop. 7.4] shows that

$$
\Gamma_{\Phi}\left(D_{\varepsilon e}\right)=1+\frac{p(p-4)}{4} \varepsilon^{2}+o\left(\varepsilon^{2}\right),
$$

as $\varepsilon>0$ tends to $0+$. Hence for $\varepsilon>0$ sufficiently small, we will have $\Gamma_{\Phi}\left(D_{\varepsilon e}\right)>$ $1=\Gamma_{\Phi}\left(D_{0}\right)$. Now, if $0 \in D_{w}$ then let $f_{w}$ be a univalent function from $D$ onto $D_{w}$ with $f_{w}(0)=0$. By the remarks following Problem 1, because of the univalence of the $f_{w}$ we will have

$$
\Lambda_{\Phi}\left(f_{\varepsilon e}\right)=\Gamma_{\Phi}\left(D_{\varepsilon e}\right)>\Gamma_{\Phi}\left(D_{0}\right)=\Lambda_{\Phi}(z),
$$

for sufficiently small $\varepsilon>0$.

Perhaps some insight could be gained into Problem 4 by numerical solutions to the necessary condition for extremality given in $\S 3$.

Finally, we have the following criterion for upper semicontinuity in the case of a function $\Phi$ which is sufficiently strongly dominated by a function $\Psi$ with $\Lambda_{\Psi}$ bounded. As usual, write $y^{+}=\max (y, 0)$.

Theorem 5. Let $\left\{f_{n}\right\}$ be a sequence of measurable, almost everywhere finite functions on $I$, converging in measure to an almost everywhere finite function $f$. Let $\Phi$ be a finite-valued upper semicontinuous function on $[0, \infty)$, and let $\Psi$ be a nonnegative Borel-measurable function on $[0, \infty)$ such that $\Lambda_{\Psi}$ is uniformly bounded on $\left\{f_{n}\right\} \cup\{f\}$. Suppose that

$$
\Phi^{+}(t)=o(\Psi(t))
$$

as $t \rightarrow \infty$. Then $\lim \sup _{n \rightarrow \infty} \Lambda_{\Phi}\left(f_{n}\right) \leq \Lambda_{\Phi}(f)$.

The proof of this result will be given in $\S 5$. Theorem 5 immediately yields the following corollary.

Corollary 2. If $|\Phi(t)|=o(\Psi(t))$ where $\Phi$ is continuous and $\Lambda_{\Psi}$ is bounded on some set $B$ of functions on a finite measure space $I$, then $\Lambda_{\Phi}$ is sequentially continuous with respect to the topology of convergence in measure on this set.

Our Theorem 1 on the impossibility of sharpening the Chang-Marshall inequality is a simple corollary of this, and we prove it as follows.

Proof of Theorem 1. Suppose $\lim _{t \rightarrow \infty} \phi(t)=\infty$ and suppose $\Lambda_{\Phi}$ is bounded on the collection $B=\left\{B_{a}: a \in D\right\}$ of the Beurling functions. Then $e^{t^{2}}=o(\Phi(t))$ as $t \rightarrow \infty$, so that by Corollary 2 we would have $f \mapsto \int_{\mathbb{T}} e^{|f|^{2}}$ weak continuous at 0 in $B \cup\{0\}$. But this is false as Cima and Matheson show [8, end of proof of Thm. 1]. For completeness, however, we give here a different proof of this lack of weak continuity at zero on $B \cup\{0\}$ by using the sharpness of Beurling's estimate. For, if $E_{M}=E_{M}\left(B_{a}\right)$ with $M$ and $a$ related by (0.8), then we may apply (0.7) to see that

$$
\int_{\mathbb{T}} e^{\left|B_{a}\right|^{2}} \geq e^{M^{2}} E_{M}+\left(1-E_{M}\right) \geq c+\left(1-E_{M}\right),
$$

for an absolute constant $c>0$. Now, by Beurling's estimate (0.6) we have $E_{M} \rightarrow 0$ as $M \rightarrow \infty$ (or, equivalently, as $|a| \rightarrow 1-$ ), so that $\liminf _{|a| \rightarrow 1-} \int_{\mathbb{T}} e^{\left|B_{a}\right|^{2}} \geq 1+c$, while it is easy to see (from the Taylor series expansions) that $B_{a} \rightarrow 0$ weakly as $a \rightarrow$ 
$1-$, and of course $\int_{\mathbb{T}} e^{0}=1$. Therefore, the Chang-Marshall functional restricted to $B \cup\{0\}$ fails to be upper semicontinuous at zero, and so $\lim _{t \rightarrow \infty} \phi(t)=\infty$ is impossible. In particular, it is not upper semicontinuous at $0 \in \mathfrak{B}$.

Cima and Matheson [8] have shown by an ad hoc method that the ChangMarshall functional $\Lambda_{\Phi_{1}}$ is weak continuous on $\mathfrak{B} \backslash\{0\}$. This fact implies that to show the existence of an extremal function for $\Lambda_{\Phi_{1}}$ it would suffice, for example, to show that if $f_{n} \rightarrow 0$ weakly in $\mathfrak{B}$ then $\lim \sup _{n \rightarrow \infty} \Lambda_{\Phi_{1}}\left(f_{n}\right) \leq e=\Lambda_{\Phi_{1}}(z)$, where $z$ denotes the identity function.

For $0 \leq r<1$, let

$$
\mathfrak{S}(r)=\left\{f \in \mathfrak{D}: r<\|f\|_{\mathfrak{D}} \leq 1\right\},
$$

be a solid half-open spherical shell in $\mathfrak{D}$. The following result then generalizes Cima and Matheson's result concerning weak continuity away from zero. We shall give the proof in $\S 5$. The proof is not very difficult and does not depend on the ChangMarshall inequality. It is quite similar to the proof of (0.9) attributed to J. Garnett in [7]. We note in passing that $\mathfrak{S}(r)$ is not weakly closed, its weak closure being all of $\mathfrak{B}$.

Theorem 6. Fix $0 \leq r<1$. Suppose that $\Phi:[0, \infty) \rightarrow \mathbb{R}$ is upper semicontinuous, and that we have $\Phi^{+}(t)=O\left(e^{\alpha t^{2}}\right)$ for every $\alpha>1 /\left(1-r^{2}\right)$. Then $\Lambda_{\Phi}$ is weak upper semicontinuous on $\mathfrak{S}(r)$.

Remark. If we take $\mathfrak{S}(r, R)=\left\{f \in \mathfrak{D}: r<\|f\|_{\mathfrak{D}} \leq R\right\}$, then we can scale the above result to see that if $\Phi$ is finite-valued and upper semicontinuous with $\Phi^{+}(t)=O\left(e^{\alpha t^{2}}\right)$ for every $\alpha>1 /\left(R^{2}-r^{2}\right)$, then $\Lambda_{\Phi}$ is weak upper semicontinuous on $\mathfrak{S}(r, R)$.

The following result, which is more general than the result of Cima and Matheson mentioned above, follows immediately from Theorem 6 , as $\mathfrak{B} \backslash\{0\}=\mathfrak{S}(0)$.

Corollary 3. Suppose that $\Phi:[0, \infty) \rightarrow \mathbb{R}$ is upper semicontinuous, and that we have $\Phi^{+}(t)=O\left(e^{\alpha t^{2}}\right)$ for every $\alpha>1$. Then $\Lambda_{\Phi}$ is weak upper semicontinuous on $\mathfrak{B} \backslash\{0\}$.

Remark. The result clearly applies to $\Phi_{1}(t)=e^{t^{2}}$. Thus the difficult part of the Chang-Marshall inequality (just as of the problem of determining whether it has an extremal function) is to handle sequences converging weakly to zero.

Finally we have the following result which we give so as not to leave the reader completely in the dark about the question of norm continuity. While we had defined the $\Lambda_{\Phi}$ only on $\mathfrak{B}$, we can define them on $\mathfrak{D}$ as well. In particular, this result shows that the Chang-Marshall functionals $\Lambda_{\Phi_{\alpha}}$ are norm continuous on all of $\mathfrak{D}$ for every $\alpha<\infty$.

Corollary 4. Suppose that $\Phi:[0, \infty) \rightarrow \mathbb{R}$ is upper semicontinuous, and that for some $\alpha \in \mathbb{R}$ we have $\Phi^{+}(t)=O\left(e^{\alpha t^{2}}\right)$. Then $\Lambda_{\Phi}$ is upper semicontinuous on $\mathfrak{D}$ with respect to the norm topology.

Proof. Possibly replacing $\alpha$ by something larger, we may assume that $\Phi^{+}(t)=$ $o\left(e^{\alpha t^{2}}\right)$. Let $f_{n} \rightarrow f$ in $\mathfrak{D}$-norm. There are two cases to be considered. If $f \equiv 0$, then choose $\delta>0$ such that $\alpha \delta<1$. For $n$ sufficiently large we will have $\left\|f_{n}\right\|_{\mathfrak{D}}^{2} \leq \delta$. Now $\Lambda_{\Phi_{\eta}}$, where $\Phi_{\eta}(t)=e^{\eta t^{2}}$, is bounded on $\mathfrak{B}$ if $\eta \leq 1$ (and if $\eta<1$ then this does not require the Chang-Marshall inequality but only Beurling's estimate), so that 
by scaling, $\Lambda_{\Phi_{\alpha}}$ is bounded on $\mathfrak{B}(\sqrt{\delta}) \stackrel{\text { def }}{=}\left\{f \in \mathfrak{D}:\|f\|_{\mathfrak{D}}^{2} \leq \delta\right\}$. Then using the fact that $\Phi^{+}(t)=o\left(e^{\alpha t^{2}}\right)$ and applying Theorem 5 to the sequence $\left\{f_{n}\right\}_{n=N}^{\infty}$ where $N$ is chosen so that $\left\|f_{n}\right\|_{\mathfrak{D}}^{2} \leq \delta$ whenever $n \geq N$, we find that $\limsup _{n \rightarrow \infty} \Lambda_{\Phi}\left(f_{n}\right) \leq$ $\Lambda_{\Phi}(f)$. (We have, of course, used the fact that if $f_{n} \rightarrow f$ in norm, then it also converges weakly.)

On the other hand if $\|f\|_{\mathfrak{D}}>0$, then let $M=\|f\|_{\mathfrak{D}}^{2}$. We have $\left\|f_{n}\right\|_{\mathfrak{D}}^{2} \rightarrow M$. Fix $\varepsilon>0$ to be chosen later, supposing for now only that $\varepsilon<M$. For $n$ large enough we will have $M-\varepsilon<\|f\|_{\mathfrak{D}}^{2} \leq M+\varepsilon$. Let $r=M-\varepsilon$ and $R=M+\varepsilon$. Then for sufficiently large $n$ we have $f_{n} \in \mathfrak{S}(r, R)$. Note that $R^{2}-r^{2} \rightarrow 0$ as $\varepsilon \rightarrow 0$, so we may choose $\varepsilon$ sufficiently small that $\alpha \leq 1 /\left(R^{2}-r^{2}\right)$. Then $\beta>\alpha$ whenever $\beta>1 /\left(R^{2}-r^{2}\right)$, so that for $\beta>1 /\left(R^{2}-r^{2}\right)$ we have $\Phi^{+}(t)=o\left(e^{\beta t^{2}}\right)$. Using the scaled version of Theorem 6 , we see that we must have $\limsup _{n \rightarrow \infty} \Lambda_{\Phi}\left(f_{n}\right) \leq \Lambda_{\Phi}(f)$.

\section{Generalizations of the EXistence And Semicontinuity theorems}

2.1. Other Dirichlet spaces. We introduce two families of spaces sharing many of the properties of the holomorphic Dirichlet space $\mathfrak{D}$. Our first family consists of the complex $M$-supported Dirichlet spaces. For $M \subseteq \mathbb{Z}_{*} \stackrel{\text { def }}{=} \mathbb{Z} \backslash\{0\}$, we define

$$
\begin{gathered}
\mathfrak{D}_{M}=\{f: f \text { is complex valued and harmonic on } D \text {, such that } \\
\left.\qquad\|f\|_{\mathfrak{D}}^{2}<\infty \text { and } \forall k \in \mathbb{Z} \backslash M . \hat{f}(k)=0\right\},
\end{gathered}
$$

where

$$
\|f\|_{\mathfrak{D}}^{2}=\frac{1}{2 \pi} \iint_{D}|\nabla f(x, y)|^{2} d x d y,
$$

and $\hat{f}(k)$ is the $k$ th Fourier coefficient of the boundary values of $f$ on $\mathbb{T}$. By the Cauchy-Riemann equations, we see that if $f$ is holomorphic on $D$ then $|\nabla f(x, y)|^{2}=$ $2\left|f^{\prime}(x+i y)\right|^{2}$, so that (2.1) agrees with (0.1). In fact, we easily see that for $f \in \mathfrak{D}_{M}$ we have

$$
\|f\|_{\mathfrak{D}}^{2}=\sum_{k \in M}|k \| \hat{f}(k)|^{2},
$$

and just as for the holomorphic case we may define an inner product and obtain a Hilbert space. Note also that our requirement that $M \subseteq \mathbb{Z}_{*}$ implies that every $f \in \mathfrak{D}_{M}$ has mean zero on $\mathbb{T}$. The particularly interesting cases are the complex harmonic Dirichlet space $\mathfrak{d} \stackrel{\text { def }}{=} \mathfrak{D}_{\mathbb{Z}_{*}}$ and the holomorphic Dirichlet space $\mathfrak{D}_{\mathbb{Z}^{+}}=\mathfrak{D}$, where $\mathbb{Z}^{+}=\{1,2,3, \ldots\}$.

Our second family consists of the real $(M, N)$-supported Dirichlet spaces, which for $M, N \subseteq \mathbb{Z}^{+}$are defined by

$$
\begin{gathered}
\mathfrak{D}_{M, N}=\left\{f: f \text { is real valued and harmonic on } D, \text { such that }\|f\|_{\mathfrak{D}}^{2}<\infty,\right. \\
\left.\forall k \in \mathbb{Z}_{0}^{+} \backslash M . \mathfrak{c}_{k}(f)=0 \text { and } \forall k \in \mathbb{Z}^{+} \backslash N . \mathfrak{s}_{k}(f)=0\right\},
\end{gathered}
$$

where $Z_{0}^{+}=\mathbb{Z}^{+} \cup\{0\}$, and where

$$
f\left(e^{i \theta}\right) \sim \sum_{k=0}^{\infty} \mathfrak{c}_{k}(f) \cos k \theta+\sum_{k=1}^{\infty} \mathfrak{s}_{k}(f) \sin k \theta .
$$


As before, $\|f\|_{\mathfrak{D}}^{2}$ is defined by (2.1). We can easily verify that for $f \in \mathfrak{D}_{M, N}$ we have

$$
\|f\|_{\mathfrak{D}}^{2}=\frac{1}{2}\left(\sum_{k \in M} k\left|\mathfrak{c}_{k}(f)\right|^{2}+\sum_{k \in N} k\left|\mathfrak{s}_{k}(f)\right|^{2}\right) .
$$

Note that $\mathfrak{D}_{M, N}$ is a real Hilbert space with the obvious inner product. Note also that every $f \in \mathfrak{D}_{M, N}$ has mean zero on $\mathbb{T}$. The particularly interesting case is the real harmonic Dirichlet space $\mathfrak{d}_{\mathbb{R}}$ def $=\mathfrak{D}_{\mathbb{Z}^{+}, \mathbb{Z}^{+}}$.

Now define $\mathfrak{B}_{M}$ and $\mathfrak{B}_{M, N}$ to be the closed unit balls of $\mathfrak{D}_{M}$ and $\mathfrak{D}_{M, N}$, respectively. For conciseness, write $\mathfrak{b}=\mathfrak{B}_{\mathbb{Z}_{*}}$ for the closed unit ball of $\mathfrak{d}$ and $\mathfrak{b}_{\mathbb{R}}=\mathfrak{B}_{\mathbb{Z}^{+}, \mathbb{Z}^{+}}$ for the closed unit ball of $\mathfrak{d}_{\mathbb{R}}$. Now, suppose $f_{n} \rightarrow f$ weakly in one of the $\mathfrak{B}_{M}$ or $\mathfrak{B}_{M, N}$. Then, we can write $f_{n}=g_{n}+\bar{h}_{n}$ and $f=g+\bar{h}$, where $g$ and $h$ as well as the $g_{n}$ and $h_{n}$ are all found in $\mathfrak{B}$, with $g_{n} \rightarrow g$ and $h_{n} \rightarrow h$ weakly in $\mathfrak{B}$. This follows from (2.2) and (2.3), together with (0.2). Then, $g_{n} \rightarrow g$ and $h_{n} \rightarrow h$ in measure on $\mathbb{T}$ by the properties of weak convergence in $\mathfrak{B}$, and it follows that $f_{n} \rightarrow f$ in measure on $\mathbb{T}$. Thus the general measure-theoretic results of the previous section work just as well for $\mathfrak{B}_{M}$ and $\mathfrak{B}_{M, N}$ as for $\mathfrak{B}$.

However there is a problem when we come to the theorems which deal specifically with the Dirichlet space, i.e., those which employ the Beurling estimate or the Chang-Marshall inequality. Thus, we now take some time to discuss the analogues of these results in $\mathfrak{b}$. Note that any boundedness results we prove on $\mathfrak{b}$ immediately give results on $\mathfrak{B}_{M}$ and $\mathfrak{B}_{M, N}$, since, clearly, $\mathfrak{B}_{M} \subseteq \mathfrak{b}$ for every $M \subseteq \mathbb{Z}_{*}$, and $\mathfrak{B}_{M, N} \subseteq \mathfrak{b}_{\mathbb{R}} \subset \mathfrak{b}$ for every $M, N \subseteq \mathbb{Z}^{+}$.

Chang and Marshall [7] note that their inequality remains valid in the real harmonic Dirichlet space. (Note, however, that our notation is somewhat different from theirs. While we have chosen to define our $\mathfrak{D}_{M}$ and $\mathfrak{D}_{M, N}$ spaces so that the norms on their intersections coincide, Chang and Marshall choose a norm on $\mathfrak{d}_{\mathbb{R}}$ such that the critical exponent for their inequality remains 1.) In our notation we have the following easy harmonic analogues of Beurling's and Chang-Marshall's results. Note that the critical exponent has now become $1 / 2$ instead of 1 . In $\S 5$ we show that both results follow from the Beurling and Chang-Marshall results for the holomorphic Dirichlet space $\mathfrak{D}$.

Theorem 7. Suppose $f \in \mathfrak{b}$. Then

$$
E_{M}(f) \leq 2 e^{1-M^{2} / 2},
$$

for every $M \geq 0$.

Conversely, if we assume that $M \geq \sqrt{\pi}$ and that $M$ and a are related by (0.8), and if we put $K=M-\pi / M$, then

$$
E_{K}\left(\sqrt{2} \operatorname{Re} B_{a}\right) \geq c e^{-K^{2} / 2}
$$

for some absolute constant $c>0$.

Note. Of course it is easy to verify using (2.3) and (0.2) that

$$
\|\operatorname{Re} f\|_{\mathfrak{D}}=(1 / \sqrt{2})\|f\|_{\mathfrak{D}},
$$

for $f \in \mathfrak{D}$. Thus $\left\|\sqrt{2} \operatorname{Re} B_{a}\right\|_{\mathfrak{D}}=1$. Note also that the constant $c$ may be different from the one in (0.6). Finally, note that (0.9) remains true in our new case. The proof is the same as Garnett's proof of the original (0.9), given in [7], but using (2.4) in place of (0.6). 
Theorem 8. If $\Phi_{\alpha}(t)=e^{\alpha t^{2}}$ and $\alpha \leq 1 / 2$, then $\Lambda_{\Phi_{\alpha}}$ is bounded on $\mathfrak{b}$. Conversely, if $\phi(t) \rightarrow \infty$ and $\Phi(t)=\phi(t) e^{t^{2} / 2}$, then $\Lambda_{\Phi}$ fails to be bounded on $\left\{(1 / \sqrt{2}) \operatorname{Re} B_{a}\right.$ : $a \in D\}$.

Remark. Chang and Marshall [7] noticed that $\Lambda_{\Phi_{1 / 2}}$ is bounded on $\mathfrak{b}_{\mathbb{R}}$. Of course $\mathfrak{b}_{\mathbb{R}} \subset \mathfrak{b}$.

We thus see that the critical exponent for Chang-Marshall type results on $\mathfrak{b}$ and $\mathfrak{b}_{\mathbb{R}}$ is $1 / 2$, and that $\Lambda_{\Phi_{1 / 2}}$ remains bounded. This leads to the following questions.

Open Problem 5. Let $B$ be one of the $\mathfrak{B}_{M}$ or $\mathfrak{B}_{M, N}$. We call $\alpha_{0}$ the ChangMarshall critical exponent of $B$ if $\alpha_{0}$ is the supremum of numbers $\alpha$ such that $\Lambda_{\Phi_{\alpha}}$ is bounded on $B$. What are the Chang-Marshall critical exponents of $\mathfrak{B}_{M}$ and $\mathfrak{B}_{M, N}$ for different choices of $M$ and $N$ ? Does $\Lambda_{\Phi_{\alpha_{0}}}$ always remain bounded when $\alpha_{0}$ is the Chang-Marshall critical exponent, assuming that $\alpha_{0}<\infty$ ?

Theorem 8 shows that $1 / 2 \leq \alpha_{0}$ for every such $B$, since each such $B$ is contained in $\mathfrak{b}$. Actually upon deeper investigation of the above question, it might turn out that for some choices of $M$, or of $M$ and $N$, it is more natural to consider functions other than the $e^{\alpha t^{2}}$ to get an inequality of Chang-Marshall type which is maximally sharp in the sense of Theorem 1. It should also be noted that if $M$ and $N$ are finite sets or even if $\sum_{n \in M} \frac{1}{|n|}$ and $\sum_{n \in N} \frac{1}{|n|}$ converge, then $\alpha_{0}=\infty$, since in this case $\mathfrak{D}_{M}$ and $\mathfrak{D}_{M, N}$ embed into $C(\mathbb{T})$.

By an easy adaptation of Beurling's proof of (0.6), it can be shown that if $B=\mathfrak{B}_{M}$, then

$$
\alpha_{0} \geq \liminf _{r \rightarrow 1-} \frac{\log \frac{1}{1-r}}{\sum_{n \in M} \frac{1}{|n|} r^{|n|}}
$$

To prove this, it suffices to show that if $\beta$ is strictly less than the right hand side of this, then we have

$$
E_{K}(f) \leq C e^{-\beta K^{2}},
$$

for $f \in \mathfrak{B}_{M}$ and $K$ finite, where $C$ is a constant possibly depending on $\beta$ and $M$. For, given this, it follows from the analogue of $(0.10)$ that $\Lambda_{\Phi_{\gamma}}$ is bounded on $\mathfrak{B}_{M}$ for every $\gamma<\beta$. But it is not difficult to adapt the proof of Beurling's estimate in [3, pp. 34-37] to yield (2.7). To do this, one first notes that by Chebyshev's inequality, $E_{K}(f) \leq K^{-2}\|f\|_{2}^{2} \leq K^{-2}\|f\|_{\mathfrak{D}}^{2} \leq K^{-2}$ if $f \in \mathfrak{B}_{M}$. This implies that $E_{K}(f) \rightarrow 0$ uniformly in $f \in \mathfrak{B}_{M}$ as $K \rightarrow \infty$. One then sets $1-(r / R)^{2}=E_{K}(f) / e$, as in the last sentence of Beurling's proof, and considers at first only $K$ sufficiently large that

$$
\frac{\log \frac{1}{1-\frac{r^{2}}{R^{2}}}}{\sum_{n \in M} \frac{1}{|n|} \frac{r^{2|n|}}{R^{2|n|}}} \geq \beta .
$$

This can be done, as these inequalities must hold for $r / R$ sufficiently close to 1 since we have chosen $\beta$ to be strictly lower than the right hand side of (2.6) and since $1-(r / R)^{2} \rightarrow 1$ as $K \rightarrow \infty$. For such large $K$ one can then easily adapt Beurling's proof to obtain (2.7) with $C=e$. For smaller $K$ one can use the trivial estimate $E_{K}(f) \leq 1$, which may possibly force one to increase the constant $C$. 
Note that if $M=\mathbb{Z}_{*}$ so that $\mathfrak{B}_{M}=\mathfrak{b}$, we then have

$$
\frac{\log \frac{1}{1-r}}{\sum_{n \in \mathbb{Z}_{*}} \frac{1}{|n|} r^{|n|}}=1 / 2,
$$

for every $r$, and arguing much as above we can in fact obtain (2.7) with $\beta=1 / 2$ and $C=e$, which is an improvement on the constant in (2.4).

We may now easily translate all the theorems and corollaries of the previous section from $\mathfrak{B}$ to $\mathfrak{B}_{M}$ and $\mathfrak{B}_{M, N}$. The only thing that needs to be done is to scale the exponents to reflect the fact that the Chang-Marshall critical exponent need no longer be 1. A representative example of this is given by the following generalization of Theorem 6 .

For $0 \leq r<1$, let

$$
\mathfrak{S}_{M}(r)=\left\{f \in \mathfrak{D}_{M}: r<\|f\|_{\mathfrak{D}} \leq 1\right\},
$$

be a solid half-open spherical shell in $\mathfrak{D}_{M}$, and similarly define the shell $\mathfrak{S}_{M, N}(r)$ in $\mathfrak{D}_{M, N}$.

Theorem 9. Fix $0 \leq r<1$. Let $\mathfrak{S}^{\prime}(r)$ be one of the $\mathfrak{S}_{M}$ or $\mathfrak{S}_{M, N}$, and let $\alpha_{0}$ be the Chang-Marshall critical exponent of the corresponding $\mathfrak{B}_{M}$ or $\mathfrak{B}_{M, N}$. Suppose that $\Phi:[0, \infty) \rightarrow \mathbb{R}$ is upper semicontinuous. If $\alpha_{0}<\infty$, then suppose that we have $\Phi^{+}(t)=O\left(e^{\alpha t^{2}}\right)$ for every $\alpha>\alpha_{0} /\left(1-r^{2}\right)$, and if $\alpha_{0}=\infty$, then suppose that $\Phi^{+}(t)=O\left(e^{\alpha t^{2}}\right)$ for some finite $\alpha$. Then $\Lambda_{\Phi}$ is weak upper semicontinuous on $\mathfrak{S}^{\prime}(r)$. In particular, if we have $\Phi^{+}(t)=O\left(e^{\alpha t^{2}}\right)$ for every $\alpha>1 /\left(2\left(1-r^{2}\right)\right)$, then $\Lambda_{\Phi}$ is weak upper semicontinuous on every $\mathfrak{S}_{M}$ and $\mathfrak{S}_{M, N}$.

The "in particular" clause follows from the fact that $\alpha_{0} \geq 1 / 2$. Note that the case $\alpha_{0}=\infty$ is in fact trivial since in that case if $\Phi^{+}(t)=O\left(e^{\alpha t^{2}}\right)$ for some $\alpha<\infty$ then $\Lambda_{\Phi_{\alpha+1}}$ is bounded on $B$, and $\Phi^{+}(t)=o\left(e^{(\alpha+1) t^{2}}\right)$, so that by Theorem 5 we have $\Lambda_{\Phi}$ weak upper semicontinuous on all of $B$.

All the other results of $\S 1$ can be analogously translated.

2.2. Removing the radial symmetry. Finally we would like to note that all the results of $\S 1$, together with the generalizations mentioned in $\S 2.1$, can be routinely generalized to deal with functionals of the form

$$
f \mapsto \int_{\mathbb{T}}(\Phi \circ f),
$$

where $\Phi: \mathbb{C} \rightarrow \mathbb{R}$ and the conditions on the growth of $\Phi(t)$ as $t \rightarrow \infty$ are replaced by conditions on the growth of $\Phi(w)$ as $|w| \rightarrow \infty$ uniformly in $\arg w$. In fact, the results of $\S 1$ which concerned functions on an arbitrary finite measure space $I$ (and not specifically the functions from $\mathfrak{D}$ ) can be easily generalized to allow the functions to take values in $\mathbb{R}^{n}$ and to consider the functionals

$$
f \mapsto \int_{I}(\Phi \circ f),
$$

where $\Phi: \mathbb{R}^{n} \rightarrow \mathbb{R}$. 


\section{Regularity of extremal FunCtions AND}

\section{A NECESSARY CONDITION FOR EXTREMALITY}

The following necessary condition for extremality is of some interest on its own, and is needed in the proof of our result on the regularity of extremal functions. It is proved in $\S 5$.

Theorem 10. Write $\Psi(t)=\Phi(\sqrt{t})$. Assume that $\Psi$ is differentiable on $(0, \infty)$, with $\left|\Psi^{\prime}(t)\right| \leq c e^{C t}$ for some finite constants $c$ and $C$, and every $t>0$. Suppose $f \in \mathfrak{B}$ is an extremal function for $\Lambda_{\Phi}$. Write

$$
S_{\Psi}(f)=\int_{\mathbb{T}}|f|^{2} \Psi^{\prime}\left(|f|^{2}\right) .
$$

Then if $S_{\Psi}(f)$ vanishes, it follows that $f \Psi^{\prime}\left(|f|^{2}\right)$ vanishes almost everywhere on $\mathbb{T}$. Assuming that $S_{\Psi}(f)$ does not vanish, we have $f^{\prime} \in H^{2}$ and

$$
S_{\Psi}(f) z f^{\prime}=\mathcal{P}_{0}\left(f \Psi^{\prime}\left(|f|^{2}\right)\right)
$$

on $\mathbb{T}$, where $z$ stands for the identity function on $\mathbb{T}$, and $\mathcal{P}_{0}$ is the orthogonal projection from $L^{2}(\mathbb{T})$ to $H_{0}^{2}(\mathbb{T}) \stackrel{\text { def }}{=}\left\{f \in H^{2}(\mathbb{T}): \hat{f}(0)=0\right\}$.

Moreover, if we also have $\|f\|_{\mathfrak{D}}<1$, then $S_{\Psi}(f)$ must vanish.

Remarks. The functional $\Lambda_{\Phi}$ makes sense on $\mathfrak{B}$ since the hypotheses guarantee that $|\Psi(t)| \leq c C^{-1}\left(e^{C t}-1\right)+|\Psi(0)|$, which, together with (0.9), guarantees the finiteness of $\Lambda_{\Phi}(f)$ for every $f \in \mathfrak{B}$ (indeed, for every $f \in \mathfrak{D}$ ). Similarly, the right hand side of (3.1) and the integral defining $S_{\Psi}(f)$ make sense because of $(0.9)$ and our assumption on the size of $\left|\Psi^{\prime}\right|$.

Also, we note that the identity function always satisfies (3.1) even though it may not be extremal. (For example, it will not be extremal if $\Phi(t)=t^{2 n}$ and $n$ is sufficiently large.)

The interested reader may, of course, translate the conditions on $\Psi$ into conditions on $\Phi$, but they are perhaps more naturally stated for $\Psi^{\prime}$. This will be even more true in the next theorem.

If $\Psi^{\prime}$ (or, equivalently, $\Phi^{\prime}$ ) does not vanish on $(0, \infty)$, and $S_{\Psi}(f)=0$, then the vanishing of $f \Psi^{\prime}\left(|f|^{2}\right)$ on $\mathbb{T}$ implies that $f \equiv 0$, and (3.1) trivially continues to hold.

The criterion (3.1) was shown by Andreev and Matheson (see [1, Cor. 3 and remarks following it]) in the special cases of the Chang-Marshall functions $\Phi_{\alpha}(t)=$ $e^{\alpha t^{2}}$ and of the functions $\Phi(t)=t^{2 n}$, under the auxiliary assumption that $f^{\prime} \in$ $H^{1}$. Our result above shows that this assumption will automatically be satisfied whenever $f$ is extremal and $\Phi^{\prime}$ does not vanish on $(0, \infty)$.

Note that for any $\Phi$ the functions $f$ of the form $f(z)=a z^{n}$ where $n|a|^{2}=1$ (in particular this includes the identity function) provide solutions to (3.1). However, in general they may not be the only solutions. For instance, if $\Phi(t)=t^{2 n}$ where $2 n>4$, then an extremal exists as noted before, and clearly the hypotheses of Theorem 10 are satisfied. But we have seen that the extremal in that case is not the identity function. Nor can it be of the form $f(z)=a z^{n}$ with $n|a|^{2}=1$, since for any such function we have $\Lambda_{\Phi}(f) \leq \Lambda_{\Phi}(z)$, where $z$ indicates the identity function.

If one could prove that for all $\alpha<1$ sufficiently close to 1 all solutions $f$ of (3.1) are of the form $f(z)=a z^{n}$ with $n|a|^{2}=1$, then it would follow that 
$\sup _{g \in \mathfrak{B}} \Lambda_{\Phi_{\alpha}}(g) \leq e^{\alpha}$ for $\alpha<1$ sufficiently close to 1 , and so by a limiting argument we would conclude that $\Lambda_{\Phi_{1}}(g) \leq e=\Lambda_{\Phi_{1}}(z)$, which would give an affirmative answer to Problem 2.

Numerical solutions to (3.1) might lead to a better understanding of the extremal functions for $\Lambda_{\Phi}$.

Open Problem 6. Investigate numerical algorithms for solving pseudodifferential equations on $\mathbb{T}$ of the form

$$
z f^{\prime}=\mathcal{P}_{0}\left(f \psi\left(|f|^{2}\right)\right)
$$

where $f$ is the boundary value of an analytic function and $\psi$ a sufficiently nice real-valued function.

The following question is related to Problem 1.

Open Problem 7. If $\Phi\left(e^{t}\right)$ is convex in $t$ and $\Phi^{\prime}(x)>0$ for every $x>0$, then does it follow from (3.1) that $f$ is automatically univalent? If so, then must it also be starlike?

Finally, as a corollary to Theorem 10, we obtain our result on the regularity of extremal functions. The proof is also given in $\S 5$.

Theorem 11. Write $\Psi(t)=\Phi(\sqrt{t})$. Fix an integer $n \geq 0$. Suppose that $\Psi$ is $n$ times differentiable on $(0, \infty)$. If $n>0$ then assume further that $\Psi^{(n)}$ is Lipschitz on bounded subintervals of $[0, \infty)$, and, if $n=0$, then assume that $\Psi$ is differentiable on $(0, \infty)$. Also assume that $0<\left|\Psi^{\prime}(t)\right| \leq c e^{C t}$ for some finite constants $c$ and $C$, and for every $t>0$. Finally assume that $f \in \mathfrak{B}$ is an extremal function for $\Lambda_{\Phi}$. Then $f$ is $n$ times continuously differentiable on $\mathbb{T}$. Furthermore, $f^{(n)}$ is absolutely continuous on $\mathbb{T}$, and in fact $f^{(n+1)} \in \mathrm{BMOA}$ so that $f^{(n)} \in \Lambda^{*}$.

Remarks. The Zygmund class $\Lambda^{*}$ is the set of functions $g$ on $\mathbb{T}$ such that for every $\theta \in[0,2 \pi)$ we have $F\left(e^{i(\theta+h)}\right)+F\left(e^{i(\theta-h)}\right)-2 F\left(e^{i \theta}\right)=O(h)$.

Of course if $\Lambda_{\Phi}$ has no extremal functions, then the content of the theorem as stated is null. Note, however, that as the proof will show, the conclusion of Theorem 11 holds not only for extremal functions $f$, but indeed for any functions $f$ satisfying the conclusion of Theorem 10 with $S_{\Psi}(f) \neq 0$.

Theorem 2, which we stated in the Introduction, follows from Theorem 11.

\section{Generalizations of Results from Section 3}

Let $\Phi: \mathbb{R}^{k} \rightarrow \mathbb{R}$ where $k=2$ or $k=1$. When convenient, we shall make the identification $\mathbb{C}=\mathbb{R}^{2}$. We consider the functionals

$$
\Lambda_{\Phi}(f)=\int_{\mathbb{T}}(\Phi \circ f),
$$

on the various $\mathfrak{B}_{M}$ and $\mathfrak{B}_{M, N}$ defined in $\S 2.1$, with $k=2$ in the case of the $\mathfrak{B}_{M}$ and $k=1$ in the case of the $\mathfrak{B}_{M, N}$. Note that if $\Psi:[0, \infty) \rightarrow \mathbb{R}$ then the previously defined $\Lambda_{\Psi}$ agrees with the newly defined $\Lambda_{\Phi}$ if we take $\Phi(z)=\Psi(|z|)$.

The following result will generalize Theorem 10. For $M \subseteq \mathbb{Z}$ we can define $L_{M}^{p}(\mathbb{T})$ to be the space of those complex-valued functions on $\mathbb{T}$ lying in $L^{p}(\mathbb{T})$ whose Fourier transforms are supported on $M$. Similarly, for $M \subseteq \mathbb{Z}^{+}$and $N \subseteq \mathbb{Z}_{0}^{+}$, we can define 
$L_{M, N}^{p}(\mathbb{T})$ to be the space of those real-valued functions on $\mathbb{T}$ lying in $L^{p}(\mathbb{T})$ whose Fourier cosine and sine transforms are supported on $M$ and $N$, respectively. Write $\mathcal{P}_{M}$ and $\mathcal{P}_{M, N}$ for the orthogonal projections from $L^{2}$ to $L_{M}^{2}$ and $L_{M, N}^{2}$, respectively. Write $\mathcal{H}$ for the isometry $L_{0}^{2}(\mathbb{T}) \rightarrow L_{0}^{2}(\mathbb{T})$ defined by $z^{n} \mapsto \operatorname{sgn}(n) z^{n}$ for $n \neq 0$.

In the foregoing, $(\nabla \Phi)(f)$ will indicate $\nabla \Phi$ evaluated at $f$, where $\nabla \Phi$ is the gradient if $k=2$ and the derivative if $k=1$. We denote the euclidean inner product of $x, y \in \mathbb{R}^{k}$ by $\langle x, y\rangle$. Of course if $k=1$ then $\langle x, y\rangle=x y$.

Theorem 12. Let $B$ be one of the $\mathfrak{B}_{M}$ or $\mathfrak{B}_{M, N}$. If $B$ is a $\mathfrak{B}_{M}$, then let $k=2$, and if $B$ is a $\mathfrak{B}_{M, N}$, then let $k=1$. Let $\Phi: \mathbb{R}^{k} \rightarrow \mathbb{R}$. Assume that $\Lambda_{\Phi}$ is defined on $B$, and that $\Phi$ has first partials $\Phi_{, j}$ everywhere on $\mathbb{R}^{k}$, satisfying $\left|\Phi_{, j}(z)\right| \leq c e^{C|z|^{2}}$, for every $z \in \mathbb{R}^{k}$, where $1 \leq j \leq k$, and $c$ and $C$ are any finite constants. Suppose $f \in B$ is an extremal function for $\Lambda_{\Phi}$. Write

$$
Q_{\Phi}(f)=\int_{\mathbb{T}}\langle f,(\nabla \Phi)(f)\rangle .
$$

Assuming that $Q_{\Phi}(f)$ does not vanish, we have $\frac{d f\left(e^{i \theta}\right)}{d \theta} \in L^{2}(\mathbb{T})$ and

$$
-i Q_{\Phi}(f) \frac{d f\left(e^{i \theta}\right)}{d \theta}=\mathcal{P}^{\prime} \mathcal{H}((\nabla \Phi)(f))
$$

on $\mathbb{T}$, where $\mathcal{P}^{\prime}$ is either $\mathcal{P}_{M}$ or $\mathcal{P}_{M, N}$, corresponding to the choice of $B$ as $\mathfrak{B}_{M}$ or $\mathfrak{B}_{M, N}$, respectively.

Moreover, if we also have $\|f\|_{\mathfrak{D}}<1$ then $Q_{\Phi}(f)$ must vanish.

Remarks. The proof of the last sentence of the theorem is an easily justified differentiation of the function $r \mapsto \Lambda_{\Phi}(r f)$ at $r=1$. In the case of $B=\mathfrak{B}_{M}$, one proves the rest of the theorem by using exactly the same variational methods as in the proof of Theorem 10. The only conceivable difficulty is where the proof asserts the boundedness of $\mathcal{P}_{0}$ by means of the M. Riesz theorem. However, the proof only used the $L^{2}$ case of the M. Riesz theorem, and it is still trivially true that $\mathcal{P}_{M}$ is bounded from $L^{2}$ to $L_{M}^{2}$. It should also be noted that at the point at which we had previously asserted that if $f$ is extremal then so is $-i f$, one must modify the remark to note that if $f$ is extremal for $\Lambda_{\Phi(\cdot)}$ then $-i f$ is extremal for $\Lambda_{\Phi(i(\cdot))}$.

In the case of $B=\mathfrak{B}_{M, N}$, we simply need to use two slightly different variations, namely

$$
f \mapsto \frac{f+\lambda C_{m}}{\left\|f+\lambda C_{m}\right\|_{\mathfrak{D}}}
$$

for $m \in M$, and

$$
f \mapsto \frac{f+\lambda S_{n}}{\left\|f+\lambda S_{n}\right\|_{\mathfrak{D}}},
$$

for $n \in N$, where $C_{m}\left(e^{i \theta}\right)=\cos m \theta$ and $S_{m}\left(e^{i \theta}\right)=\sin m \theta$. The rest of the proof adapts.

The careful reader will note that Theorem 12 is not a complete generalization of Theorem 10. First of all, Theorem 10 had a discussion of what happens when $S_{\Psi}(f)$ vanishes. We cannot reproduce this, because the proof of it was based on analyticity. Secondly, Theorem 10, when translated into the language of Theorem 12, did not 
require the first partials of $\Phi$ to exist at zero. We may explain this as follows. The proof of Theorem 10 which was adapted to yield Theorem 12 still goes through if we only assume that, first of all, $\Phi_{, j}(z)$ exists on $\mathbb{R}^{k} \backslash E$ for some set $E$ such that $\left|\left\{e^{i \theta}: f\left(e^{i \theta}\right) \in E\right\}\right|=0$, and that, secondly, $\Phi$ is Lipschitz on compact subsets of some $\varepsilon$-neighbourhood of the image of $f$. In the holomorphic case of Theorem 12 we simply took $E=\{0\}$ and noted that if $|f| \in E$ on a set of positive measure, then $f \equiv 0$ and the results are trivial.

Definition. We say that $\Phi: \mathbb{R}^{k} \rightarrow \mathbb{R}$ satisfies our strict smooth monotonicity condition if $\Phi$ is Lipschitz on compact subsets of $\mathbb{R}^{k}$ and has

$$
\left.\frac{d \Phi(r z)}{d r}\right|_{r=1}>0
$$

for every $z \neq 0$ for which this derivative is defined.

The primary purpose of this definition is the remark that if $\Phi$ satisfies our strict smooth monotonicity condition, and $Q_{\Phi}(f)$ vanishes, then $f$ must vanish a.e. on $\mathbb{T}$. We now obtain the following regularity result. Theorem 3 follows immediately from it.

Theorem 13. Fix $n \geq 0$. Let $\Phi: \mathbb{R}^{k} \rightarrow \mathbb{R}$, for $k=2$ or $k=1$, and let $B$ be $\mathfrak{B}_{M}$ or $\mathfrak{B}_{M, N}$, if $k=2$ or $k=1$, respectively. Assume that $\Lambda_{\Phi}$ is defined on $B$, and that $\Phi$ has first partials $\Phi_{, j}$ everywhere on $\mathbb{R}^{2}$, satisfying $\left|\Phi_{, j}(z)\right| \leq c e^{C|z|^{2}}$ for every $z \in \mathbb{R}^{2}$, where $1 \leq j \leq k$ and $c$ and $C$ are any finite constants. Assume that $\Phi$ satisfies our strict smooth monotonicity condition (4.2). Suppose that all the (pure and mixed) partials of $\Phi$ of order $\leq n$ exist. If $n>0$ then assume further that all the (pure and mixed) nth order partials are Lipschitz on compact subsets of $\mathbb{R}^{2}$. Suppose $f \in B$ is an extremal function for $\Lambda_{\Phi}$. Then $f$ is $n$ times continuously differentiable on $\mathbb{T}$. Furthermore, the nth derivative of $f$ on $\mathbb{T}$ is absolutely continuous and the $(n+1)$ st derivative lies in $\mathcal{P}^{\prime}\left[L^{\infty}(\mathbb{T})\right]$, where $\mathcal{P}^{\prime}$ is as in Theorem 12. Finally, the $n$th order partial derivatives are everywhere defined on $\bar{D}$.

Remark. In the last sentence we allow derivatives with respect to polar coordinates, and in particular one sided derivatives with respect to $r$. The last sentence is a consequence of the fact that the $(n+1)$ st derivative of $f$ lies in $L^{2}(\mathbb{T})$, which allows us to split $f$ as the sum of an analytic and an antianalytic function, each of which has $(n+1)$ st derivative in $L^{2}(\mathbb{T})$, and hence each is the $n$th antiderivative of a function in the disc algebra or in the anti-analytic disc algebra, and the $n$th antiderivatives of functions from the disc algebra satisfy the last sentence of the theorem. Indeed, this sort of splitting of $f$ into analytic and antianalytic parts whenever we need to obtain an absolute continuity result allows us to modify the proof of Theorem 11 to obtain Theorem 13.

Finally we would like to make note of one more generalization. For simplicity of notation we only consider the case of functionals on $\mathfrak{B}$, but everything easily adapts to $\mathfrak{B}_{M}$ and $\mathfrak{B}_{M, N}$. Let $L$ be a closed subset of $\mathbb{C}^{l}$ for some $l \in \mathbb{Z}^{+}$, and set

$$
\mathfrak{B}^{L}=\{f \in \mathfrak{B}:(\hat{f}(1), \hat{f}(2), \ldots, \hat{f}(l)) \in L\} .
$$

Note that $\mathfrak{B}^{L}$ is weakly compact since $f \mapsto \hat{f}(j)$ is weak continuous on $\mathfrak{D}$ by $(0.3)$. Then, assuming we have the conditions of Theorem 12 satisfied with $\mathfrak{B}^{L}$ in place 
of $B$, the proof of Theorem 10 can be seen to imply that if $f$ is extremal with $\|f\|_{\mathfrak{D}}=1$ and $Q_{\Phi}(f) \neq 0$, then

$$
-i Q_{\Phi}(f) \mathcal{P}_{l}\left(z f^{\prime}\right)=\mathcal{P}_{l}((\nabla \Phi)(f)),
$$

where $\mathcal{P}_{l}$ is the orthogonal projection from $L^{2}(\mathbb{T})$ to $H_{l}^{2}(\mathbb{T}) \stackrel{\text { def }}{=}\left\{f \in H^{2}(\mathbb{T}): \hat{f}(j)=\right.$ $0,0 \leq j \leq l\}$. This is so because the proof of Theorem 10 proceeds by varying the Taylor coefficients of $f$, and in our new case we simply leave $\hat{f}(1), \ldots, \hat{f}(l)$ fixed and vary only $\hat{f}(j)$ for $j>l$.

Assume now that $\Phi$ satisfies our strict smooth monotonicity condition (4.2). Then $f \equiv 0$ if $Q_{\Phi}(f)=0$. Also, as noted in the remarks following Theorem 12, if $\|f\|_{\mathcal{D}}<1$ and $f$ is extremal, then $Q_{\Phi}(f)=0$, so that $f \equiv 0$. Of course the case where $f$ vanishes is trivial from the point of view of regularity questions.

Thus if $f \not \equiv 0$ is extremal, then (4.4) is valid, so that there is an analytic polynomial $p$ with $p(0)=0$ and degree at most $l$ such that

$$
Q_{\Phi}(f) z f^{\prime}+p=\mathcal{P}_{0}((\nabla \Phi)(f))
$$

Now the proof of Theorem 13 can be modified to use this expression in place of (4.1) since $p$ is entire and thus has all the desired regularity properties. We then obtain the following result.

Theorem 14. Let $L$ be a closed subset of $\mathbb{C}^{l}$, and let $\mathfrak{B}^{L}$ be defined by (4.3). Then Theorem 13 continues to hold if we set $k=2$ and replace $B$ by $\mathfrak{B}^{L}$ in it.

This is of particular interest in conjunction with Corollary 3 which implies that, since $\mathfrak{B}^{L}$ is weakly compact, extremal functions will exist over $\mathfrak{B}^{L}$ provided $0 \notin L$ and $|\Phi(t)|=O\left(e^{\alpha t^{2}}\right)$ for every $\alpha>1$. In particular we obtain the following result when we let $L=\{z \in \mathbb{C}:|z| \geq \delta\}$.

Corollary 5. Let $n$ be a non-negative integer and $0 \leq \alpha \leq 1$; assume that at least one of $n$ and $\alpha$ is nonzero. Put $\Phi(z)=|z|^{2 n} e^{\alpha|z|^{2}}$, and fix $\delta>0$. Then $\Lambda_{\Phi}$ is weak continuous on $\left\{f \in \mathfrak{B}:\left|f^{\prime}(0)\right| \geq \delta\right\}$, and hence achieves its maximum there. Furthermore, those functions $f \in \mathfrak{B}$ with $\left|f^{\prime}(0)\right| \geq \delta$ at which $\Lambda_{\Phi}$ achieves this maximum must lie in $C^{\infty}(\bar{D})$.

Remarks. Recall that by Theorem 1 , the functional $\Lambda_{\Phi}$ will not be bounded on $\mathfrak{B}$ if $n>0$ and $\alpha=1$, and that for $n=0$ and $\alpha=1$ it is bounded (Chang-Marshall inequality) but it is not known whether it achieves its maximum there.

\section{Proofs of more difficult Results from Sections 1,2 And 3}

\subsection{Proofs of the more difficult results from Section 1.}

Proof of Theorem 5. Dropping to a subsequence, we may assume that we have almost everywhere convergence. For, every subsequence of $\left\{f_{n}\right\}$ has an almost everywhere convergent subsubsequence, and it suffices to show that for every subsequence $f_{n_{k}}$ along which $\Lambda_{\Phi}\left(f_{n_{k}}\right)$ converges the limit is no greater than $\Lambda_{\Phi}(f)$.

Now fix $\varepsilon>0$. Let $M$ be a uniform upper bound for $\Lambda_{\Psi}$ on $\left\{f_{n}\right\} \cup\{f\}$. Since $\Phi^{+}(t)=o(\Psi(t))$ and $\Psi$ is nonnegative, we may choose a number $T$ such that 
$\Phi(t) \leq(\varepsilon / 4 M) \Psi(t)$ whenever $t \geq T$. Furthermore, we may ensure that our choice of $T$ is such that

$$
\mu\{x:|f(x)|=T\}=0
$$

and

$$
|\Phi(0)| \mu\{x:|f(x)|>T-1\} \leq \varepsilon / 4,
$$

since $f$ is almost everywhere finite.

Let $U(z)=z$ if $|z|<T$ and $U(z)=0$ if $|z| \geq T$. Then $U \circ f_{n} \rightarrow U \circ f$ almost everywhere outside the set $\{x:|f(x)|=T\}$. By (5.1) this latter set has measure zero, so we have convergence almost everywhere. Since $\Phi$ is upper semicontinuous, we also have

$$
\limsup _{n \rightarrow \infty} \Phi\left(\left|U \circ f_{n}\right|\right) \leq \Phi(|U \circ f|),
$$

almost everywhere. Now the $\left|U \circ f_{n}\right|$ are uniformly bounded by $T$, so that the $\Phi\left(\left|U \circ f_{n}\right|\right)$ are uniformly bounded by some constant $K$ independent of $n$ since $\Phi$ is bounded on the compact interval $[0, T]$, being upper semicontinuous and finite. Then using the fact that we have a finite measure space, and applying Fatou's lemma to the non-negative functions $K-\Phi\left(\left|U \circ f_{n}\right|\right)$, we find that

$$
\limsup _{n \rightarrow \infty} \Lambda_{\Phi}\left(U \circ f_{n}\right) \leq \int_{I} \limsup _{n \rightarrow \infty} \Phi\left(\left|U \circ f_{n}\right|\right) \leq \Lambda_{\Phi}(U \circ f),
$$

where the last inequality follows from (5.3).

But by the definition of $U$ we have

$$
\begin{aligned}
\Lambda_{\Phi}\left(f_{n}\right)-\Lambda_{\Phi}\left(U \circ f_{n}\right) & =\int_{\left\{x:\left|f_{n}(x)\right| \geq T\right\}}\left(\Phi\left(\left|f_{n}\right|\right)-\Phi(0)\right) \\
& \leq\left(\int_{\left\{x:\left|f_{n}(x)\right| \geq T\right\}}(\varepsilon / 4 M) \Psi\left(\left|f_{n}\right|\right)\right)-\Phi(0) \mu\left\{x:\left|f_{n}(x)\right| \geq T\right\} \\
& \leq(\varepsilon / 4 M) M-\Phi(0) \mu\left\{x:\left|f_{n}(x)\right| \geq T\right\},
\end{aligned}
$$

where in the last two inequalities we have used the choices of $T$ and $M$, respectively. Furthermore, by (5.2) and the fact that $f_{n}$ converges in measure to $f$, it follows that $\lim \sup _{n \rightarrow \infty}|\Phi(0)| \mu\left\{x:\left|f_{n}(x)\right| \geq T\right\} \leq \varepsilon / 4$. Therefore,

$$
\limsup _{n \rightarrow \infty} \Lambda_{\Phi}\left(f_{n}\right)-\Lambda_{\Phi}\left(U \circ f_{n}\right) \leq \varepsilon / 2 .
$$

In much the same way we find that

$$
\left|\Lambda_{\Phi}(f)-\Lambda_{\Phi}(U \circ f)\right| \leq \varepsilon / 2 .
$$

Then, putting (5.4), (5.5) and (5.6) together, we find that

$$
\begin{aligned}
\limsup _{n \rightarrow \infty} \Lambda_{\Phi}\left(f_{n}\right)-\Lambda_{\Phi}(f)= & \limsup _{n \rightarrow \infty}\left[\Lambda_{\Phi}\left(f_{n}\right)-\Lambda_{\Phi}\left(U \circ f_{n}\right)\right. \\
& +\Lambda_{\Phi}\left(U \circ f_{n}\right)-\Lambda_{\Phi}(U \circ f) \\
& \left.+\Lambda_{\Phi}(U \circ f)-\Lambda_{\Phi}(f)\right] \\
\leq & \varepsilon
\end{aligned}
$$


Since $\varepsilon>0$ was arbitrary, the proof is complete.

While reading the following proof, the reader may wish to bear in mind that we have promised that it would not depend on the Chang-Marshall inequality.

Proof of Theorem 6. Recall that we defined $\Phi_{\alpha}(t)=e^{\alpha t^{2}}$. By our hypotheses, for every $\alpha>1 /\left(1-r^{2}\right)$ we have $\Phi^{+}(t)=O\left(\Phi_{\alpha}(t)\right)$, as $t \rightarrow \infty$. But since $\Phi_{\beta}(t)=$ $o\left(\Phi_{\alpha}(t)\right)$ whenever $\alpha>\beta$, we have in fact $\Phi^{+}(t)=o\left(\Phi_{\alpha}(t)\right)$ for every $\alpha>1 /\left(1-r^{2}\right)$, since we may choose a $\beta \in\left(1 /\left(1-r^{2}\right), \alpha\right)$ and we will have $\Phi^{+}(t)=O\left(\Phi_{\beta}(t)\right)$.

Now let $f_{n} \rightarrow f$ weakly in $\mathfrak{S}(r)$. We must show that

$$
\limsup _{n \rightarrow \infty} \Lambda_{\Phi}\left(f_{n}\right) \leq \Lambda_{\Phi}(f) .
$$

Write $f(z)=\sum_{k=1}^{\infty} \hat{f}(k) z^{k}$. Since $\|f\|_{\mathfrak{D}}^{2}>r^{2}$ for $f \in \mathfrak{S}(r)$, by (0.2) we may choose a natural number $K$ sufficiently large that

$$
\sum_{k=1}^{K} k|\hat{f}(k)|^{2}>r^{2} .
$$

Let $h(z)=\sum_{k=1}^{K} \hat{f}(k) z^{k}$. Put $g_{n}=f_{n}-h$. We claim that $\lim \sup _{n \rightarrow \infty}\left\|g_{n}\right\|_{\mathfrak{D}}^{2}<$ $1-r^{2}$. Indeed, (0.3) shows that the weak convergence of $f_{n}$ to $f$ implies (in fact, is equivalent to) the convergence of $\hat{f}_{n}(k) \rightarrow \hat{f}(k)$ for every natural $k$. Then

$$
\begin{aligned}
\left\|g_{n}\right\|_{\mathfrak{D}}^{2} & =\sum_{k=K+1}^{\infty} k\left|\hat{f}_{n}(k)\right|^{2}+\sum_{k=1}^{K} k\left|\hat{f}_{n}(k)-\hat{f}(k)\right|^{2} \\
& =\left\|f_{n}\right\|_{\mathfrak{D}}^{2}-\sum_{k=1}^{K} k\left|\hat{f}_{n}(k)\right|^{2}+\sum_{k=1}^{K} k\left|\hat{f}_{n}(k)-\hat{f}(k)\right|^{2} \\
& \leq 1-\sum_{k=1}^{K} k\left|\hat{f}_{n}(k)\right|^{2}+\sum_{k=1}^{K} k\left|\hat{f}_{n}(k)-\hat{f}(k)\right|^{2},
\end{aligned}
$$

since $\left\|f_{n}\right\|_{\mathfrak{D}} \leq 1$. Now, since $\hat{f}_{n}(k) \rightarrow \hat{f}(k)$ for every $k$, it follows that the right hand side of the above inequality converges to $1-\sum_{k=1}^{K} k|\hat{f}(k)|^{2}=1-\|h\|_{\mathfrak{D}}^{2}<1-r^{2}$, where the last inequality is a consequence of (5.7). The claim is thus proved. Now choose $\gamma$ such that

$$
\limsup _{n \rightarrow \infty}\left\|g_{n}\right\|_{\mathfrak{D}}^{2}<\gamma<1-r^{2} .
$$

For $n$ sufficiently large we then have $g_{n} \in \mathfrak{B}(\sqrt{\gamma})=\left\{f \in \mathfrak{D}:\|f\|_{\mathfrak{D}}^{2} \leq \gamma\right\}$.

Scaling the fact that $\Phi_{\eta}$ is bounded on $\mathfrak{B}$ if $\eta<1$ (and also if $\eta=1$, but that would require the Chang-Marshall inequality which we have promised to avoid), we find that $\Phi_{\beta}$ is bounded on $\mathfrak{B}(\sqrt{\gamma})$ if $\beta \gamma<1$. Since $\gamma<1-r^{2}$, we may choose $\alpha$ such that $\alpha \gamma<1$ and $\alpha\left(1-r^{2}\right)>1$. We claim that $\Lambda_{\Phi_{\alpha}}$ is bounded on the $f_{n}$ corresponding to those $g_{n}$ which lie in $\mathfrak{B}(\sqrt{\gamma})$. Assume for now that this is proved. Then, since $\Lambda_{\Phi_{\alpha}}$ is finite on $\mathfrak{D}$ by $(0.9)$, and since $g_{n} \in \mathfrak{B}(\sqrt{\gamma})$ for sufficiently large $n$, and finally since $\alpha>1 /\left(1-r^{2}\right)$ so that $\Phi^{+}(t)=o\left(e^{\alpha t^{2}}\right)$, it follows from Theorem 5 that $\limsup _{n \rightarrow \infty} \Lambda_{\Phi}\left(f_{n}\right) \leq \Lambda_{\Phi}(f)$. 
To prove our claim, note that for every $z \in \mathbb{T}$ we have $\left|f_{n}(z)\right|=\left|g_{n}(z)+h(z)\right| \leq$ $\left|g_{n}(z)\right|+|h(z)|$, and $h$ is bounded on $\mathbb{T}$, being a polynomial. Since $\alpha \gamma<1$, we may choose $\beta>\alpha$ such that we still have $\beta \gamma<1$. Then, since $h$ is bounded, there exists a finite number $M=M\left(\|h\|_{\infty}, \alpha, \beta\right)$ such that

$$
e^{\alpha\left|f_{n}\right|^{2}} \leq e^{\alpha\left(|h|+\left|g_{n}\right|\right)^{2}} \leq M e^{\beta\left|g_{n}\right|^{2}}
$$

on $\mathbb{T}$. But we have already noted that $\Lambda_{\Phi_{\beta}}$ is bounded on $\mathfrak{B}(\sqrt{\gamma})$ when $\beta \gamma<1$, and so we see that $\Lambda_{\Phi_{\alpha}}$ must be bounded on those $f_{n}$ with $g_{n} \in \mathfrak{B}(\sqrt{\gamma})$. This completes the proof.

\subsection{Proofs of the Beurling and Chang-Marshall results for the complex harmonic Dirichlet space.}

Proof of Theorems 7 and 8. We begin by proving the first statement of both theorems. We may suppose $\|f\|_{\mathfrak{D}}=1$. Otherwise, renormalize it, which will everywhere increase $|f|$. We may also suppose $\alpha=1 / 2$ in Theorem 8. Since $f \in \mathfrak{d}$, we may write $f=g+\bar{h}$, where $g$ and $h$ are holomorphic functions from $\mathfrak{D}$. Then $1=\|f\|_{\mathfrak{D}}^{2}=\|g\|_{\mathfrak{D}}^{2}+\|h\|_{\mathfrak{D}}^{2}$, as can easily be seen from (2.2). Let $A=\|g\|_{\mathfrak{D}}^{2}$ and $B=\|h\|_{\mathfrak{D}}^{2}$. Fix $\lambda>0$, which will be chosen later. Let $S=\{\omega \in \mathbb{T}:|g(\omega)| \geq \lambda|h(\omega)|\}$, and let $S^{c}$ be its complement. For $\omega \in S$, we have

$$
|f(\omega)| \leq|g(\omega)|+|\bar{h}(\omega)| \leq|g(\omega)|+\lambda^{-1}|g(\omega)|=\left(1+\lambda^{-1}\right)|g(\omega)| .
$$

For $\omega \in S^{c}$, we similarly have

$$
|f(\omega)| \leq(1+\lambda)|h(\omega)|
$$

Now $g$ and $h$ satisfy the Beurling estimate and the Chang-Marshall inequality. Scaling the Beurling estimate we get

$$
E_{M}\left(\left(1+\lambda^{-1}\right) g\right) \leq e^{1-M^{2} /\left(A\left(1+\lambda^{-1}\right)^{2}\right)},
$$

and

$$
E_{M}((1+\lambda) h) \leq e^{1-M^{2} /\left(B(1+\lambda)^{2}\right)},
$$

since the Dirichlet norms of $g$ and $h$ are $\sqrt{A}$ and $\sqrt{B}$, respectively. Splitting $\mathbb{T}$ into $S$ and $S^{c}$, we see that we will obtain (2.4) if we can choose $\lambda>0$ so that

$$
A\left(1+\lambda^{-1}\right)^{2} \leq 2
$$

and

$$
B(1+\lambda)^{2} \leq 2
$$

From (5.8) and (5.9), we would also obtain

$$
\int_{S} e^{|f|^{2} / 2} \leq \int_{S} e^{\left|\left(1+\lambda^{-1}\right) g\right|^{2} / 2} \leq \int_{\mathbb{T}} e^{|g|^{2} / A} \leq C,
$$


since $\|g / \sqrt{A}\|_{\mathfrak{D}}=1$, and

$$
\int_{S^{c}} e^{|f|^{2} / 2} \leq \int_{S^{c}} e^{|(1+\lambda) h|^{2} / 2} \leq \int_{\mathbb{T}} e^{|h|^{2} / B} \leq C,
$$

since $\|h / \sqrt{B}\|_{\mathfrak{D}}=1$, and where $C=\sup _{F \in \mathfrak{B}} \Lambda_{\Phi_{1}}(F)$ is the constant in the ChangMarshall inequality. Adding the two expressions, we would be able to conclude that $\Lambda_{\Phi_{1 / 2}}(f) \leq 2 C$, and the proof of the first part of Theorem 8 would be complete.

Thus to complete the proofs of the first parts of the theorems, it suffices to show that we may choose $\lambda>0$ satisfying both (5.8) and (5.9). This is an elementary consequence of the facts that $A+B=1$ and $0 \leq A, B \leq 1$. If $B=0$ then it is trivially easy to find such a $\lambda$. Thus, assume $B>0$. Choose $\lambda>0$ such that $B(1+\lambda)^{2}=2$. Then (5.9) is trivial. Now,

$$
A\left(1+\lambda^{-1}\right)^{2}=(1-B)\left(1+\lambda^{-1}\right)^{2}=\lambda^{-2}(1-B)(1+\lambda)^{2}=\lambda^{-2}\left((1+\lambda)^{2}-2\right),
$$

since $B\left(1+\lambda^{2}\right)=2$, so that (5.8) is equivalent to the inequality

$$
(1+\lambda)^{2}-2 \leq 2 \lambda^{2}
$$

But this is equivalent to the inequality

$$
\lambda^{2}-2 \lambda+1 \geq 0
$$

which is certainly true for every $\lambda \in \mathbb{R}$. Thus the proof of the first parts of the theorems is complete.

Now note that by rescaling, (2.5) is equivalent to the inequality

$$
E_{M}\left(\operatorname{Re} B_{a}\right) \geq c e^{-M^{2}} .
$$

Note also that it follows directly from the definition of $B_{a}$ that if $a$ and $M$ are related by $(0.8)$, then $\left|\operatorname{Im} B_{a}\right| \leq \pi / M$, since the absolute value of the imaginary part of the chosen branch of $\log (1-\bar{a} z)$ cannot exceed $\pi$. Hence, if $\left|B_{a}(\omega)\right| \geq M$ for some $\omega \in \mathbb{T}$, then we have $\left|\operatorname{Re} B_{a}(\omega)\right| \geq M-\pi / M=K$. Thus,

$$
E_{K}\left(\operatorname{Re} B_{a}\right) \geq E_{M}\left(B_{a}\right) \geq c^{\prime} e^{-M^{2}},
$$

where the last inequality follows from (0.7) and where we have renamed the constant appearing there to $c^{\prime}$ in order to avoid confusion with the $c$ appearing in (5.10). But

$$
e^{-K^{2}}=e^{-(M-\pi / M)^{2}}=e^{-\left(M^{2}-2 \pi+\pi^{2} / M^{2}\right)}<e^{2 \pi} e^{-M^{2}} .
$$

Letting $c=e^{-2 \pi} c^{\prime}$, we see that (5.11) implies (5.10). This completes the proof of Theorem 7 .

Just as in the proof of Theorem 1, we now see that it follows that $\Lambda_{\Phi_{1 / 2}}$ is not upper semicontinuous at zero on $\{0\} \cup\left\{\sqrt{2} \operatorname{Re} B_{a}: a \in D\right\}$, and hence by Theorem 5 we cannot have $\Lambda_{\Phi}$ bounded on the $\sqrt{2} \operatorname{Re} B_{a}$ if $\Phi(t) / \Phi_{1 / 2}(t) \rightarrow \infty$ as $t \rightarrow \infty$. This completes the proof of Theorem 8 . 
5.3. Proofs of the results from Section 3. We shall use the following trivial and very classical lemma to analyze the case where $S_{\Psi}(f)=0$. We write $\bar{H}^{p}(\mathbb{T})=$ $\left\{\bar{f}: f \in H^{p}(\mathbb{T})\right\}$ for the antianalytic Hardy spaces.

Lemma 2. (see Koosis [13, p. 87]) Let $V \in H^{p}(\mathbb{T})$ or $V \in \bar{H}^{p}(\mathbb{T})$ for some $p$, $1 \leq p \leq \infty$, be real valued on $\mathbb{T}$. Then $V$ is almost everywhere constant.

Proof. Without loss of generality consider the case $V \in H^{p}(\mathbb{T})$. By [13, p. 87] we can continue $V$ analytically to all of $\mathbb{C}$ by setting $V(z)=\overline{f(1 / \bar{z})}$ for $|z|>1$. Then $V$ will be a bounded entire function, hence constant.

The careful reader will note that at several points in the following proof we would be able to assert that various functions lie in $L^{p}$ for every $p<\infty$, but we only write down that they lie in $L^{2}$ or $L^{1}$. We do this in order to make it clearer how to generalize the proof to yield Theorem 12 .

Proof of Theorem 10. We proceed by a simple variational argument. Let $f$ be extremal. Write $f(z)=\sum_{n=1}^{\infty} a_{n} z^{n}$.

Suppose first that $\|f\|_{\mathfrak{D}}=1$. Write $F(\lambda)=\left\|f+\lambda z^{n}\right\|_{\mathfrak{D}}^{2}$, where we use $z^{n}$ as a short form for the function $z \mapsto z^{n}$. Since $\|f\|_{\mathfrak{D}}=1$ while $\left\|z^{n}\right\|_{\mathfrak{D}}=\sqrt{n}$, it follows that, for $|\lambda|<1 / \sqrt{n}$, we have $F(\lambda)>0$ and $\left(f+\lambda z^{n}\right) / \sqrt{F(\lambda)}$ has unit Dirichlet norm. Suppose now that $\lambda$ is real. If $f$ is extremal, then we must have

$$
\left.\frac{d \Lambda_{\Phi}\left(\left(f+\lambda z^{n}\right) / \sqrt{F(\lambda)}\right)}{d \lambda}\right|_{\lambda=0}=0
$$

as long as this derivative exists. We shall prove this derivative exists, and compute its form.

First note that

$$
\begin{aligned}
F(\lambda) & =\left(\sum_{\substack{k=1 \\
k \neq n}}^{\infty} k\left|a_{k}\right|^{2}\right)+n\left|a_{n}+\lambda\right|^{2}=\left(\sum_{k=1}^{\infty} k\left|a_{k}\right|^{2}\right)+n\left[\left(a_{n}+\bar{a}_{n}\right) \lambda+\lambda^{2}\right] \\
& =\|f\|_{\mathfrak{D}}^{2}+n\left(2 \lambda \operatorname{Re} a_{n}+\lambda^{2}\right)=1+n\left(2 \lambda \operatorname{Re} a_{n}+\lambda^{2}\right) .
\end{aligned}
$$

Now we have $f(z)$ finite for almost every $z \in \mathbb{T}$. Furthermore, we may assume that $|f(z)| \neq 0$ for almost every $z$. For if $f$ vanishes on a set of positive measure then, being a function in $\mathfrak{D} \subset H^{1}$, it would have to vanish identically and the theorem would follow trivially. Moreover, if we restrict $\lambda$ to $[-1 /(2 \sqrt{n}), 1 /(2 \sqrt{n})]$ then $F(\lambda)$ will not vanish. Fixing $z$ such that $|f(z)|<\infty$, we then have $\Psi(\mid f(z)+$ $\left.\left.\lambda z^{n}\right|^{2} / F(\lambda)\right)$ absolutely continuous with respect to $\lambda$ on $[-1 /(2 \sqrt{n}), 1 /(2 \sqrt{n})]$. For, $\Psi$ is differentiable except possibly at zero, the derivative is bounded in a neighbourhood of zero (since $\left|\Psi^{\prime}(t)\right| \leq c e^{C t}$ ) and, if $z$ is fixed, then $\left|f(z)+\lambda z^{n}\right|^{2}$ can vanish for at most finitely many (in fact, for at most two) values of $\lambda$. Thus if 
$-1 /(2 \sqrt{n}) \leq \lambda \leq 1 /(2 \sqrt{n})$, we will have

$$
\begin{aligned}
\Delta_{\lambda}(z) \stackrel{\text { def }}{=} \frac{1}{\lambda}\left(\Psi\left(\frac{\left|f(z)+\lambda z^{n}\right|^{2}}{F(\lambda)}\right)-\Psi(|f(z)|)\right) \\
=\frac{1}{\lambda} \int_{0}^{\lambda}\left(\frac{d}{d t} \Psi\left(\frac{\left|f(z)+t z^{n}\right|^{2}}{F(t)}\right)\right) d t \\
=\frac{1}{\lambda} \int_{0}^{\lambda} \Psi^{\prime}\left(\frac{\left|f+t z^{n}\right|^{2}}{F(t)}\right) \\
\quad \times \frac{F(t)\left(z^{n} \bar{f}+\bar{z}^{n} f+2 t|z|^{2 n}\right)-F^{\prime}(t)\left|f+t z^{n}\right|^{2}}{(F(t))^{2}} d t \\
=\frac{1}{\lambda} \int_{0}^{\lambda} \Psi^{\prime}\left(\frac{\left|f+t z^{n}\right|^{2}}{F(t)}\right) \\
\quad \times \frac{F(t)\left(2 \operatorname{Re}\left(\bar{z}^{n} f\right)+2 t\right)-n\left(2 \operatorname{Re} a_{n}+2 t\right)\left|f+t z^{n}\right|^{2}}{(F(t))^{2}} d t,
\end{aligned}
$$

by (5.13) and the fact that $|z|=1$ on $\mathbb{T}$. Of course, as long as $0<|f(z)|<\infty$ (which happens for almost every $z \in \mathbb{T}$ ), if we let $\lambda \rightarrow 0$ then this will converge to

$$
\left.\delta(z) \stackrel{\text { def }}{=} \frac{d}{d \lambda}\right|_{\lambda=0} \Psi\left(\frac{\left|f(z)+\lambda z^{n}\right|^{2}}{F(\lambda)}\right)=2\left(\operatorname{Re}\left(\bar{z}^{n} f\right)-n \operatorname{Re} a_{n}|f|^{2}\right) \Psi^{\prime}\left(|f(z)|^{2}\right),
$$

where we have used the fact that $F(0)=\|f\|_{\mathfrak{D}}^{2}=1$. We wish to conclude that

$$
\lim _{\lambda \rightarrow 0} \int_{\mathbb{T}} \Delta_{\lambda}=\int_{\mathbb{T}} \delta
$$

and to this end we will dominate $\Delta_{\lambda}$ by an $L^{1}(\mathbb{T})$ function not depending on $\lambda$.

Now if $-1 /(2 \sqrt{n}) \leq \lambda \leq 1 /(2 \sqrt{n})$, then $1 / 4 \leq F(\lambda) \leq 9 / 4$, as can be easily verified. Thus, making simple estimates in (5.14) and using the assumption that $\left|\Psi^{\prime}(y)\right| \leq c e^{C y}$, we obtain, for $-1 /(2 \sqrt{n}) \leq \lambda \leq 1 /(2 \sqrt{n})$ and almost every $z \in \mathbb{T}$,

$$
\left|\Delta_{\lambda}\right| \leq K e^{K|f(z)|^{2}}\left(|f|+1+(n+1)(|f|+1)^{2}\right),
$$

for some finite constant $K$ depending only on $c$ and $C$. But by (0.9), the right hand side of the last expression is integrable on $\mathbb{T}$. Applying Lebesgue's dominated convergence theorem we find that (5.16) holds, so that (5.12) makes sense and we have

$$
\begin{aligned}
0 & =\left.\frac{d \Lambda_{\Phi}\left(\left(f+\lambda z^{n}\right) / \sqrt{F(\lambda)}\right)}{d \lambda}\right|_{\lambda=0}=\lim _{\lambda \rightarrow 0} \int_{\mathbb{T}} \Delta_{\lambda}=\int_{\mathbb{T}} \delta \\
& =\int_{\mathbb{T}} 2\left(\operatorname{Re}\left(\bar{z}^{n} f\right)-n \operatorname{Re} a_{n}|f|^{2}\right) \Psi^{\prime}\left(|f|^{2}\right),
\end{aligned}
$$

by (5.15). But if $f$ is extremal, then so is $-i f$, since $|f|=|-i f|$ and the Dirichlet norms are also the same. Applying (5.17) to - if then yields the same expression but with $\operatorname{Im}\left(\bar{z}^{n} f\right)$ and $\operatorname{Im} a_{n}$ in place of $\operatorname{Re}\left(\bar{z}^{n} f\right)$ and $\operatorname{Re} a_{n}$, respectively. Multiplying 
the new expression by $i$, adding to (5.17), and dividing everything by two, we find that

$$
\int_{\mathbb{T}}\left(\bar{z}^{n} \cdot f \Psi^{\prime}\left(|f|^{2}\right)\right)=n a_{n} \int_{\mathbb{T}}|f|^{2} \Psi^{\prime}\left(|f|^{2}\right)=n a_{n} S_{\Psi}(f),
$$

for every positive integer $n$.

Assume first that $S_{\Psi}(f)$ does not vanish. Then (5.18) would immediately yield (3.1) if we knew that $z f^{\prime}$ had boundary values whose positive Fourier coefficients were $\left\{n a_{n}\right\}$. This would follow if we knew that $f^{\prime} \in H^{1}$, but unfortunately we do not a priori know this, and so we must proceed more carefully.

Let $G(z)=f(z) \Psi^{\prime}\left(|f(z)|^{2}\right)$ on $\mathbb{T}$. By (0.9) and the hypothesis that $\left|\Psi^{\prime}(y)\right| \leq$ $c e^{C y}$, we have $G \in L^{2}(\mathbb{T})$. Hence $\mathcal{P}_{0}(G) \in H^{2}(\mathbb{T})$. But we see from (5.18) that the $n$th positive Fourier coefficient of $G$ is $S_{\Psi}(f) n a_{n}$, which is also the $n$th Taylor coefficient of $S_{\Psi}(f) z f^{\prime}$. Now $\mathcal{P}_{0}(G)$ extends to a holomorphic function with Taylor coefficients equal to its positive Fourier coefficients, and the positive Fourier coefficients of $\mathcal{P}_{0}(G)$ must of course match those of $G$ and these match the Taylor coefficients of $S_{\Psi}(f) z f^{\prime}$. It follows that $S_{\Psi}(f) z f^{\prime}=\mathcal{P}_{0}(G)$, which is precisely the equation (3.1). Furthermore, $f^{\prime} \in H^{2}(\mathbb{T})$ since $\mathcal{P}_{0}(G) \in H^{2}(\mathbb{T})$.

Now if $S_{\Psi}(f)$ vanishes, then, by (5.18), the positive Fourier coefficients of the function $f \Psi^{\prime}\left(|f|^{2}\right) \in L^{2}(\mathbb{T})$ must vanish. But this implies that $f \Psi^{\prime}\left(|f|^{2}\right)$ is the boundary value of an antianalytic function, call it $\bar{h}$. By (0.9) and the inequality $\Psi^{\prime}(y) \leq c e^{C y}, \bar{h}$ lies in $\bar{H}^{2}(\mathbb{T})$. Therefore, $|f|^{2} \Psi^{\prime}\left(|f|^{2}\right)=\bar{h} \cdot \bar{f}$ is also the boundary value of an antianalytic function from $\bar{H}^{1}(\mathbb{T})$, since $\bar{f}$ is antianalytic and $f \in \mathfrak{D} \subset$ $H^{2}$. Applying Lemma 2, we see that $|f|^{2} \Psi^{\prime}\left(|f|^{2}\right)$ is almost everywhere constant on $\mathbb{T}$. But since $0=S_{\Psi}(f)$ equals the integral of this constant about $\mathbb{T}$, it follows that in fact $|f|^{2} \Psi^{\prime}\left(|f|^{2}\right)=0$ almost everywhere on $\mathbb{T}$, so that $f \Psi^{\prime}\left(|f|^{2}\right)=0$ almost everywhere on $\mathbb{T}$ as desired.

The only remaining thing to do is to consider the case where $\|f\|_{\mathfrak{D}}<1$. In this case, for $\lambda$ sufficiently small we still have $f+\lambda z^{n} \in \mathfrak{B}$, so that we may use a simpler variation. Much as before, but with the calculations being somewhat simpler, we find that the condition

$$
\left.\frac{d \Lambda_{\Phi}\left(f+\lambda z^{n}\right)}{d \lambda}\right|_{\lambda=0}=0
$$

makes sense, and applied to $f$ and $-i f$ leads to the condition

$$
\int_{\mathbb{T}}\left(\bar{z}^{n} \cdot \Psi^{\prime}\left(|f|^{2}\right) f\right)=0
$$

for every positive integer $n$. As before, this implies that $f \Psi^{\prime}\left(|f|^{2}\right)$ is the boundary value of an antianalytic function, say $\bar{h}$, which as before will have to be in $\bar{H}^{2}$. Therefore, $|f|^{2} \Psi^{\prime}\left(|f|^{2}\right)$ is the boundary value of $\bar{h} \cdot \bar{f} \in \bar{H}^{1}$, so that its integral about $\mathbb{T}$ must equal $\bar{h}(0) \bar{f}(0)=0$. But its integral about $\mathbb{T}$ is just $S_{\Psi}(f)$. Thus, $S_{\Psi}(f)$ vanishes and we may complete our argument in the same way as was done in the case of $\|f\|_{\mathfrak{D}}=1$, above, but using (5.19) in place of (5.18).

We now recall the following well-known result which is crucial to the proof of our regularity theorem, though in fact we only use the easy case $p=2$ in our work. 
Lemma 3. (Privalov; see [9, Thm. 3.11]) Let $f^{\prime} \in H^{p}$ for a holomorphic function $f$ on $D$ and $p \geq 1$. Then $f$ is absolutely continuous on $\mathbb{T}$ and continuous on $\bar{D}$, and $f^{\prime}$ has the boundary values $f^{\prime}\left(e^{i \theta}\right)=-i e^{-i \theta} \frac{d f\left(e^{i \theta}\right)}{d \theta}$.

We also need the following simple result, which we list here for ease of reference and to establish a convention which we will use in our proof of Theorem 11.

Lemma 4. Let $F$ be a Lipschitz function and $G$ absolutely continuous. Then $F \circ G$ is absolutely continuous and $(F \circ G)^{\prime}=\left(F^{\prime} \circ G\right) G^{\prime}$ almost everywhere, with the convention that $\left(F^{\prime}(G(x)) G^{\prime}(x)=0\right.$ whenever $G^{\prime}(x)=0$, whether $F$ is differentiable at $G(x)$ or not.

Given this, we proceed to the proof of our regularity theorem. The main tool in the proof will be a qualitative analysis of condition (3.1). The careful reader will note that at some points in the proof we take some care to conclude that certain quantities are in $L^{2}$, and only use the fact that other quantities are in $L^{2}$, whereas it may be slightly simpler to replace all the $L^{2}$ conditions by the condition $\bigcap_{p<\infty} L^{p}$, and to use the full M. Riesz theorem. This would allow us not to bother with keeping track of the degrees of the arguments of the polynomial $p_{k+1}$. However, we choose to argue in $L^{2}$ because the M. Riesz theorem is trivial for the $L^{2}$ case and generalizes to $L_{M}^{2}$ for arbitrary $M \subseteq \mathbb{Z}$, so that it will be possible to adapt our proof to obtain Theorem 13.

Proof of Theorem 11. First note that for every $k \leq n$ the function $\Psi^{(k)}$ is bounded on the intervals $[0, A]$ for every finite $A$. This follows by $(n-k)$ integrations of the function $\Psi^{(n)}$, which is Lipschitz there.

We may also assume that $S_{\Psi}(f) \neq 0$. For if it does equal zero, then $f \Psi^{\prime}\left(|f|^{2}\right)$ vanishes on $\mathbb{T}$. But $\Psi^{\prime}$ can only vanish at zero, so we see that $f \equiv 0$ in this case, and the theorem is trivial then.

We note that (3.1) can be written in the form

$$
S z f^{\prime}=\mathcal{P}_{0}\left[p\left(f, \bar{f} ; \Psi^{\prime}\left(|f|^{2}\right)\right],\right.
$$

where $p$ is a polynomial in $2+1$ variables and $S=S_{\Psi}(f)$. The basic idea is to differentiate this expression repeatedly. Write

$$
\Delta g\left(e^{i \theta}\right)=-i \frac{d g\left(e^{i \theta}\right)}{d \theta}
$$

for a function $g$ on $\mathbb{T}$. Note that $\Delta z^{n}=n z^{n}$. If $g$ is holomorphic on $D$ and $g^{\prime} \in H^{1}$, then $\Delta g=z g^{\prime}$ by Lemma 3 .

Thus we can rewrite (5.20) as

$$
S \Delta f=\mathcal{P}_{0}\left[p\left(f, \bar{f} ; \Psi^{\prime}\left(|f|^{2}\right)\right)\right] .
$$

The main thing to do now is to prove that our hypotheses in effect allow us to commute $\Delta$ with $\mathcal{P}_{0}$ the right number of times.

We shall proceed iteratively. For the convenience of the reader, however, we first outline the idea of the proof in a less formal way in the case where $\Phi \in C^{\infty}[0, \infty)$. In that case we must show that $f \in C^{\infty}(\mathbb{T})$. By Theorem 10 and Lemma 3 we have $\Delta f=z f^{\prime} \in H^{2}$ and $f$ is absolute continuous on $\mathbb{T}$. Moreover,

$$
S \Delta f=F_{1},
$$


where $F_{1}=p\left(f, \bar{f} ; \Psi^{\prime}\left(|f|^{2}\right)\right)$. Now,

$$
\Delta F_{1}=p_{2}\left(z, \bar{z}, f, \bar{f}, f^{\prime}, \overline{f^{\prime}} ; \Psi^{\prime}\left(|f|^{2}\right), \Psi^{\prime \prime}\left(|f|^{2}\right)\right),
$$

for some polynomial $p_{2}$. Moreover, the right hand side of (5.22) is only linear in $f^{\prime}$ and $\overline{f^{\prime}}$ (i.e., $f^{\prime}$ and $\overline{f^{\prime}}$ in it are never multiplied together nor are they raised to a power bigger than 1). The absolute continuity of $f$ implies that $F_{1} \stackrel{\text { def }}{=} p\left(f, \bar{f} ; \Psi^{\prime}\left(|f|^{2}\right)\right)$ is absolutely continuous on $\mathbb{T}$, since $p$ is a polynomial. Together with an integration by parts and the fact that $S \Delta f=\mathcal{P}_{0} F_{1}$, this shows that the positive Fourier coefficients of $\Delta F_{1}$ coincide with the positive Fourier coefficients of $S \Delta^{2} f$ considered as a distribution on $\mathbb{T}$. But by (5.22) since $f^{\prime} \in H^{2}$ it follows that $\Delta F_{1} \in L^{2}(\mathbb{T})$ (here we have used the fact that the right side of (5.22) is only linear in $f^{\prime}$ and $\overline{f^{\prime}}$ ). Thus, $S \Delta^{2} f \in L^{2}(\mathbb{T})$ and

$$
S \Delta^{2} f=\mathcal{P}_{0} F_{2},
$$

where $F_{2}$ denotes the right hand side of (5.22). From the fact that $\Delta^{2} f \in L^{2}(\mathbb{T})$, it follows (using Lemma 3 ) that $f^{\prime}$ is absolutely continuous on $\mathbb{T}$ and that $f^{\prime \prime} \in H^{2}$. Thus $F_{2}$ is absolutely continuous on $\mathbb{T}$. Applying the above method one more time we conclude that $\Delta F_{2}$ is a polynomial in

$$
z, \bar{z}, f, \bar{f}, f^{\prime}, \overline{f^{\prime}}, f^{\prime \prime}, \overline{f^{\prime \prime}}, \Psi^{\prime}\left(|f|^{2}\right), \Psi^{\prime \prime}\left(|f|^{2}\right), \Psi^{\prime \prime \prime}\left(|f|^{2}\right),
$$

and that

$$
S \Delta^{3} f=\mathcal{P}_{0} \Delta F_{2} \in L^{2}(\mathbb{T}),
$$

so that $f^{\prime \prime \prime} \in H^{2}(\mathbb{T})$. Iterating we conclude that $f^{(k)} \in H^{2}(\mathbb{T})$ for all natural $k$, which implies that $f \in C^{\infty}(\mathbb{T})$ as desired.

We now return to the general case and proceed more rigorously. For the sake of our iteration, suppose that on $\mathbb{T}$ we have

$$
\begin{array}{r}
S \Delta^{k} f=\mathcal{P}_{0}\left[p _ { k } \left(z, \bar{z}, f, \bar{f}, f^{\prime}, \overline{f^{\prime}}, \ldots, f^{(k-1)}, \overline{f^{(k-1)}}\right.\right. \\
\left.\left.\Psi^{\prime}\left(|f|^{2}\right), \Psi^{\prime \prime}\left(|f|^{2}\right), \ldots, \Psi^{(k)}\left(|f|^{2}\right)\right)\right],
\end{array}
$$

with $k \leq n$, with $p_{k}$ a polynomial in $2(k+1)+k$ variables, and with $\Delta^{j} f \in H^{2}$ for every $0 \leq j \leq k$. By Theorem 10 and (5.21), we have this for $k=1$.

Using the fact that $\Delta g=z g^{\prime}$, we easily see that for any natural $j$ we can write (in the sense of power series)

$$
z^{j} f^{(j)}=\sum_{l=0}^{j} a_{j, l} \Delta^{l} f
$$

for some set of universal constants $a_{j, l}$. Since $\Delta^{j} f \in H^{2}$ for every $0 \leq j \leq k$, it follows that likewise $f^{(j)} \in H^{2}$ whenever $0 \leq j \leq k$. Thus by Lemma 3 we have that $f^{(j)}$ is absolutely continuous on $\mathbb{T}$ for $0 \leq j \leq k-1$. Since sums and products of absolutely continuous functions are absolutely continuous, and since $\Psi^{(j)}$ for $0 \leq j \leq k$ is Lipschitz on bounded subintervals of $[0, \infty)$ while $f$ is absolutely 
continuous on $\mathbb{T}$ so that $\Psi^{(j)}\left(|f|^{2}\right)$ must be absolutely continuous on $\mathbb{T}$ by Lemma 4 , it follows that

$$
\begin{array}{r}
F_{k} \stackrel{\text { def }}{=} p_{k}\left(z, \bar{z}, f, \bar{f}, f^{\prime}, \overline{f^{\prime}}, \ldots, f^{(k-1)}, \overline{f^{(k-1)}}\right. \\
\left.\Psi^{\prime}\left(|f|^{2}\right), \Psi^{\prime \prime}\left(|f|^{2}\right), \ldots, \Psi^{(k)}\left(|f|^{2}\right)\right)
\end{array}
$$

is absolutely continuous on $\mathbb{T}$. But, in the presence of absolute continuity, we may use integration by parts to see that for every positive integer $m$,

$$
\begin{aligned}
\int_{0}^{2 \pi} e^{-i m \theta} \Delta F_{k}\left(e^{i \theta}\right) d \theta & =m \int_{0}^{2 \pi} e^{-i m \theta} F_{k}\left(e^{i \theta}\right) d \theta \\
& =S m \int_{0}^{2 \pi} e^{-i m \theta} \Delta^{k} f\left(e^{i \theta}\right) d \theta,
\end{aligned}
$$

where we have applied (5.23) to obtain the last equality.

Now it is easy to see from (5.24) and the definition of $\Delta$ that

$$
\begin{aligned}
& \Delta F_{k}=p_{k+1}\left(z, \bar{z}, f, \bar{f}, f^{\prime}, \overline{f^{\prime}}, \ldots, f^{(k)}, \overline{f^{(k)}}\right. \\
&\left.\Psi^{\prime}\left(|f|^{2}\right), \Psi^{\prime \prime}\left(|f|^{2}\right), \ldots, \Psi^{(k+1)}\left(|f|^{2}\right)\right)
\end{aligned}
$$

for some polynomial $p_{k+1}$ in $2(k+1)+(k+1)$ variables, such that $f^{(k)}$ and $\overline{f^{(k)}}$ are not raised to any power greater than one nor are they multiplied together on the right side of (5.26) (i.e., fixing all arguments other than the $(2 k+1)$ st and $(2 k+2)$ nd in $p_{k+1}$ we obtain something of degree one), and where we have used the convention of Lemma 4 at points $z$ of $\mathbb{T}$ where $\Psi^{(k+1)}\left(|f(z)|^{2}\right)$ is undefined. This convention may be necessary if $k=n$ and $n>0$, since then we only know that $\Psi^{(k+1)}$ exists almost everywhere, and the set of points $z$ of $\mathbb{T}$ at which $\Psi^{(k+1)}\left(|f|^{2}(z)\right)$ is undefined may well have positive measure, since after all $|f|$ is free to be constant (as long as this constant is not zero) on a subset of $\mathbb{T}$ with positive measure, even if $f$ itself is not everywhere constant in $D$.

Since we know that $f$ is absolutely continuous on $\mathbb{T}$, it must be bounded there. As noted at the beginning of the proof, the hypotheses of the theorem imply that $\Psi^{(j)}$ is bounded everywhere on $\left[0,\|f\|_{\infty}^{2}\right]$ for $j \leq n$. This is also obviously true for $j=n+1$ if $n=0$ by our condition on the size of $\left|\Psi^{\prime}\right|$. In the case where $j=k=n+1$ and $n>0$, we note that the derivative of the function $\Psi^{(n)}$, which is Lipschitz on $\left[0,\|f\|_{\infty}^{2}\right]$, is bounded by the Lipschitz constant wherever it exists. Moreover, for almost every $z \in \mathbb{T}$ such that $|f(z)|^{2}$ falls into the exceptional set where $\Psi^{(n)}$ is not defined, we have, following the convention of Lemma 4, some factor equal to zero multiplying the $\Psi^{(n)}(|f(z)|)$ in the right hand side of (5.26). Finally, since $f^{(k)}$ is in $H^{2}$, from Lemma 3 we conclude that all arguments of $p_{k+1}$ in (5.26) are bounded, except possibly for $f^{(k)}$ and $\overline{f^{(k)}}$. But these latter two arguments are never multiplied together, nor are they ever raised to any power, so we see that since they lie in $L^{2}(\mathbb{T})$, it follows that in fact the right hand side of (5.26) must lie in $L^{2}(\mathbb{T})$. Thus, $\mathcal{P}_{0}\left(\Delta F_{k}\right) \in H^{2}(\mathbb{T})$, as well, by the $L^{2}$ case of the theorem of M. Riesz.

Now write $g(z)=S \Delta^{k} f(z)=\sum_{m=1}^{\infty} a_{m} z^{m}$ for $z \in D$. We have $z g^{\prime}(z)=$ $\sum_{m=1}^{\infty} m a_{m} z^{m}$ in $D$. In this notation, (5.25) tells us that for $m$ positive, the $m$ th 
Fourier coefficient of $\Delta F_{k}$ on $\mathbb{T}$ is $m a_{m}$, and since $\mathcal{P}_{0}\left(\Delta F_{k}\right) \in H^{2}(\mathbb{T})$, it follows as in the proof of Theorem 10 that we must have $z g^{\prime}=\mathcal{P}_{0}\left(\Delta F_{k}\right)$. But $z g^{\prime}=S \Delta^{k+1} f$, so that we obtain

$$
\begin{aligned}
& S \Delta^{k+1} f=\mathcal{P}_{0}\left[p _ { k + 1 } \left(z, \bar{z}, f, \bar{f}, f^{\prime}, \overline{f^{\prime}}, \ldots, f^{(k)}, \overline{f^{(k)}}\right.\right. \\
&\left.\left.\Psi^{\prime}\left(|f|^{2}\right), \Psi^{\prime \prime}\left(|f|^{2}\right), \ldots, \Psi^{(k+1)}\left(|f|^{2}\right)\right)\right],
\end{aligned}
$$

with $\Delta^{k+1} f=S^{-1} z g^{\prime} \in H^{2}$ since $S \neq 0$. But this was precisely what was needed for the iteration to continue.

Thus iterating, we see that $\Delta^{k} f \in H^{2}$ for each $k \leq n+1$. As argued before, it follows that $f^{(n+1)} \in H^{2}$, and so by Lemma 3 we have $f^{(n)}$ absolutely continuous on $\mathbb{T}$, and of course then $f$ must be $n$ times differentiable, as desired. Finally, since $f^{(n)}$ must be bounded, being absolutely continuous, and on the bounded interval $\left[0,\|f\|_{\infty}^{2}\right]$ we have $\Psi^{(n+1)}$ bounded wherever defined, it follows from (5.26) that $\Delta F_{n}$ must lie in $L^{\infty}(\mathbb{T})$ (where as before we use the convention of Lemma 4) so that by (5.27) it follows that $\Delta^{n+1} f \in$ BMOA, and so $f^{(n+1)} \in$ BMOA, by the argument which we have used twice before in order to pass from estimates on the $\Delta^{j} f$ to ones on the $f^{(j)}$. But, BMOA is contained in the Bloch space, and a derivative $g^{\prime}$ of a holomorphic function $g$ is in the Bloch space if and only if $g \in \Lambda^{*}$ (see, e.g., [22, vol. I, p. 163]), so that $f^{(n)} \in \Lambda^{*}$.

\section{ACKNOWLEDGEMENTS}

The authors would like to thank the referee for a number of helpful suggestions with regard to the presentation of the paper, especially in the case of the proof of Theorem 11. The second author would like to thank Professor Albert Baernstein II for drawing his attention to the work of Sakai [20].

\section{REFERENCES}

1. Valentin V. Andreev and Alec Matheson, Extremal functions and the Chang-Marshall inequality, Pacific J. Math. 162 (1994), 233-246. MR 95f:30051

2. Albert Baernstein II, Integral means, univalent functions and circular symmetrization, Acta Math. 133 (1974), 139-169. MR 54:5456

3. Arne Beurling, Études sur un problème de majoration, Thèse pour le doctorat, Almqvist \& Wiksell, Uppsala, 1933.

4. F. F. Bonsall, Boundedness of Hankel matrices, J. London Math. Soc. (2) 29 (1984), 289-300. MR 85f: 47030

5. D. L. Burkholder, Exit times of Brownian motion, harmonic majorization, and Hardy spaces, Advances in Math. 26 (1977), 182-205. MR 57:14163

6. L. Carleson and S.-Y. A. Chang, On the existence of an extremal function for an inequality of J. Moser, Bull. Sc. Math. (2 ${ }^{\mathrm{e}}$ série) 110 (1986), 113-127. MR 88f:46070

7. S.-Y. A. Chang and D. E. Marshall, On a sharp inequality concerning the Dirichlet integral, Amer. J. Math. 107 (1985), 1015-1033. MR 87a:30055

8. Joseph Cima and Alec Matheson, A nonlinear functional on the Dirichlet space, J. Math. Anal. Appl. 191 (1995), 380-401. CMP 1995:10

9. Peter L. Duren, Theory of $H^{p}$ spaces, Academic Press, New York, 1970. MR 42:3552

10. M. Essén, Sharp estimates of uniform harmonic majorants in the plane, Ark. Mat. 25 (1987), 15-28. MR 89b:30024

11. M. Essén, K. Haliste, J. L. Lewis and D. F. Shea, Harmonic majorization and classical analysis, J. London Math. Soc. (2) 32 (1985), 506-520. MR 87f:30012

12. John B. Garnett, Bounded analytic functions, Academic Press, London and San Diego, 1981. MR 83g:30037 
13. Paul Koosis, Introduction to $H^{p}$ spaces, with an appendix on Wolff's proof of the corona theorem, London Math. Soc. Lecture Note Series, Vol. 40, Cambridge Univ. Press, Cambridge, 1980. MR 81c:30062

14. Moshe Marcus, Transformations of domains in the plane and applications in the theory of functions, Pacific J. Math. 14 (1964), 613-626. MR 29:2382

15. D. E. Marshall, A new proof of a sharp inequality concerning the Dirichlet integral, Ark. Mat. 27 (1989), 131-137. MR 90h:30097

16. J. B. McLeod and L. A. Peletier, Observations on Moser's inequality, Arch. Rational Mech. Anal. 106 (1989), 261-285. MR 90d:26029

17. J. Moser, A sharp form of an inequality by N. Trudinger, Indiana Univ. Math. J. 20 (1971), 1077-1092. MR 46:662

18. Alexander R. Pruss, Some remarks on a conjecture concerning harmonic majorants and radial rearrangement, Preprint. Available by anonymous ftp from math.ubc.ca as file/pub/pruss/ Conjecture.ps or /pub/pruss/Conjecture.dvi (1995).

19. Alexander R. Pruss, Nonexistence of maxima for perturbations of some inequalities with critical growth, Canad. Math. Bull. (to appear).

20. Makoto Sakai, Isoperimetric inequalities for the least harmonic majorant of $|x|^{p}$, Trans. Amer. Math. Soc. 299 (1987), 431-472. MR 88f:31005

21. W. T. Sledd and D. A. Stegenga, An $H^{1}$ multiplier theorem, Ark. Mat. 19 (1981), 265-270. MR 82j: 42018

22. A. Zygmund, Trigonometric Series, 2nd ed., Cambridge Univ. Press, London and New York, 1968. MR 38:4882

Department of Mathematics, Lamar University, Beaumont, Texas 77710

E-mail address: matheson@math.lamar.edu

Department of Mathematics, University of British Columbia, Vancouver, B.C., CANADA V6T $1 Z 2$

E-mail address: pruss@math.ubc.ca 